\title{
ANTICIPATIVE DIRECT TRANSFORMATIONS ON THE POISSON SPACE
}

\author{
Florent NICAISE \\ Laboratoire de mathématiques appliquées, CNRS-UMR 6620, Université Blaise Pascal, \\ 24, avenue des Landais, 63177 Aubière, France
}

Received 29 June 2001, revised 10 December 2001

ABSTRACT. - We study in this paper a new type of anticipative transformation on the Poisson space, which consist in adding and removing particles to an initial condition $\omega$ following a Poisson conditional distribution. We give some sufficient criteria ensuring that the distribution of the transformed system is absolutely continuous with respect to the initial Poisson distribution. Thanks to the independence properties of random Poisson measures, we split the transformation into an adapted part and an anticipative part, which is made of an almost surely finite number of modifications of the initial condition. The absolute continuity property for the adapted transformation is given by some ideas close to the ones used by Enchev and Stroock [4] in the context of transformations on the Wiener space. The same property for the anticipative part is solved thanks to some results coming from Picard [10]. As an application, we study an anticipative perturbation of a Lévy, $\beta$-stable isotropic process by another similar $\beta^{\prime}$-stable process and give sufficient conditions to have an absolute continuity property.

(c) 2002 Éditions scientifiques et médicales Elsevier SAS

MSC: 60H07; 60G51; 60G55

Keywords: Absolute continuity; Lévy processes; Malliavin calculus; Point processes; Random measures

RÉSUMÉ. - Nous étudions un nouveau type de transformation anticipante de l'espace de Poisson qui consiste à ajouter ou enlever certaine particules d'une condition initiale répartie suivant la mesure de Poisson. Sachant la condition initiale $\omega$, les modifications sont aléatoires, régies par une mesure de Poisson dont l'intensité est fonction de $\omega$. La question principale concerne l'absolue continuité de la loi du système transformé par rapport à la répartition de Poisson du système initial. Nous séparons de telles transformations en une partie adaptée et une partie anticipante : l'absolue continuité est obtenue pour la partie adaptée grâce à des idées semblables à celles utilisées par Enchev et Stroock [4] dans le cadre de l'espace de Wiener. La partie anticipative de la transformation est résolue grâce au calcul de Malliavin développé par Picard [10]. Nous appliquons ces résultats à létude de perturbations anticipatives d'un processus de Lévy $\beta$-stable par un autre processus de Lévy $\beta^{\prime}$-stable, donnant un critère d'absolue continuité pour de telles perturbations.

(c) 2002 Éditions scientifiques et médicales Elsevier SAS

E-mail address: nicaise@ math.univ-bpclermont.fr (F. Nicaise). 
Introduction. We first give an instance of the kind of problems we want to deal with.

Original Problem. - Let $\left.d \in \mathbb{N}^{*},\left\{\beta, \beta^{\prime}\right\} \subset\right] 0 ; 2\left[\right.$ and $(\Omega, \mathcal{F}, \mathbb{P}),\left(\Omega^{\prime}, \mathcal{F}^{\prime}, \mathbb{P}^{\prime}\right)$ two probability spaces. We consider a $\beta$-stable, isotropic Lévy process $X:[0 ; 1] \times \Omega \rightarrow \mathbb{R}^{d}$. We consider another Lévy process $X^{\prime}:[0 ; 1] \times \Omega^{\prime} \rightarrow \mathbb{R}^{d}$ isotropic, $\beta^{\prime}$-stable. Let give us a measurable mapping $\Psi: \mathbb{R}^{d} \rightarrow \mathbb{R}$. Can we find a sufficient condition on $\beta, \beta^{\prime}$ and $\Psi$ to have that the distribution of $\left\{\bar{X}_{t}=X_{t}+\Psi\left(X_{1}\right) X_{t}^{\prime}, t \in[0 ; 1]\right\}$ under $\mathbb{P} \otimes \mathbb{P}^{\prime}$ is absolutely continuous with respect to the one of $\left\{X_{t}, t \in[0 ; 1]\right\}$ under $\mathbb{P}$ ?

The main difficulty of this kind of question is that we perturbate the path of the Lévy process $X$. by adding to it the jumps of an independent Lévy $X^{\prime}$ following an anticipative way: the modification of the path $X .(\omega)$ at time $t$ does not only depend on the path $\left\{X_{s}(\omega), s \in[0 ; t]\right\}$, but depends on the terminal value of $X$.

The Brownian version of this kind of problem has been widely studied: let $\left\{B_{t}, t \in\right.$ $[0 ; 1]\}$ a standard Wiener process defined on some probability space $\left(W, \mathcal{F}_{W}, \mathbb{P}_{W}\right)$ where $W$ is the set of continuous mapping from $[0 ; 1]$ to $\mathbb{R}^{d}$ and $f:[0 ; 1] \times W \rightarrow \mathbb{R}$ some measurable mapping such that $\int_{0}^{1} f_{s}^{2}(\omega) d s<\infty \mathbb{P}_{W}$-almost surely. Let us define the process $\left\{\bar{B}_{t}, t \in[0 ; 1]\right\}$ by

$$
\bar{B}_{t}(\omega)=B_{t}(\omega)+\int_{0}^{t} f_{s}(\omega) d s
$$

In this setup, $\bar{B}: W \rightarrow W$ may be seen as a transformation of the Brownian path $B$ by adding to it the path of a finite variation process $(t, \omega) \mapsto \int_{0}^{t} f_{s}(\omega) d s$. The classical question asked in this context is: can we find sufficient conditions on $f$ to have that the distribution of the process $\bar{B}$ is absolutely continuous with respect to the one of $B$, that is,

$$
\mathbb{P}_{W} \circ \bar{B}^{-1} \ll \mathbb{P}_{W} \circ B^{-1} .
$$

This is the Brownian version of the Lévy original problem of the introduction, excepted that in this general setup, the perturbation of the path $B .(\omega)$ may depend on more than the terminal value $B_{1}(\omega)$ as it does in our Lévy setup. The positive answers to this Wiener problem are known as Girsanov results, although the first ones are due to Cameron and Martin [3] in the case of a deterministic $f$. The books of Nualart [8] and Ustunel and Zakai [12] give a good state of the art and an exhaustive bibliography concerning this topic. The study of the anticipative case, where $f$ is not adapted with respect to the filtration generated by $\left(B_{t}\right)_{t}$ needs tools such the Malliavin calculus and most of approaches are based upon a finite dimensional approximation of the infinite dimensional structure of the Wiener measure $\mathbb{P}_{W} \circ B^{-1}$.

However Enchev and Stroock initiate in [4] an original approach. They first study a flow of transformation $\tilde{B}:[0 ; 1] \times W \rightarrow W$ given by:

$$
\tilde{B}(t, \omega)(.)=B_{.}(\omega)+\int_{0}^{. \wedge t} h_{s}(\tilde{B}(s, \omega)) d s
$$


for some good process $h:[0 ; 1] \times W \rightarrow \mathbb{R}$. Notice that the transformations (1) where explicitly given, whereas the (3)-type ones are implicitly given. The (3)-type transformations were also widely studied, especially by Buckdahn (see [1], for instance) and Buckdahn and Enchev [2]. It was further developed by Ustunel and Zakai [13]. The technique used by Enchev and Stroock in [4] is to find sufficient conditions on $h$ to have

$$
\mathbb{P}_{W} \circ[\tilde{B}(1, .)]^{-1} \ll \mathbb{P}_{W} \circ B^{-1}
$$

Then they relate the (3)-type transformations with the (1)-type transformations in a certain sense, allowing them to transfer the results obtained for the first transformations to the study of (2).

The type of absolute continuity problems that we are going to study in this paper is a priori slightly different from the one exposed in the first paragraph. In this paper, we will not work on the process $X$ himself. We will explain in Section 4.3 how sufficient conditions to the Lévy original problem of the first paragraph may be deduced from the ones that we give in the different context of this paper.

In our paper, we consider a Lusin space $(U, \mathcal{U})$, that is, a measurable space homeomorphic to a measurable part of some Polish space and we equip it with a diffuse, $\sigma$-finite measure $\lambda^{-}$. We define $\Omega$ as, roughly speaking, the set integer-valued measures $(U, \mathcal{U})$ such that $\omega(\{u\}) \leqslant 1$ for every $u$, the sigma-field $\mathcal{F}$ and the Poisson probability $\mathbb{P}$ with intensity $\lambda^{-}$will be defined in Section 1 - the canonical example of Section 1 shows how to relate a Poisson space with a Lévy process. The obtained probability space $(\Omega, \mathcal{F}, \mathbb{P})$ is a Poisson space. We study in this framework some anticipative transformations consisting in, from an initial condition $\omega \in \Omega$, picking a random subset $\tilde{\omega} \subset U$ following a particular, stochastic way: we give us a process $f: U \times \Omega \rightarrow \mathbb{R}^{+}$such that $f_{u}(\omega) \leqslant 1$ if $\omega(\{u\})=1$ and pick a random subset - or cloud $-\widetilde{\omega} \subset U$ following: each particle $u$ of $\omega$ is taken in the cloud with probability $f_{u}(\omega)$, we include in the cloud the particles of a Poisson random measure on $U$ with intensity $f .(\omega) \lambda^{-}($.$) . Moreover,$ each inclusion is made independently of the other selections. Then we modify $\omega$ at each point of the cloud by adding a particle if there was not one at this point and removing the particle if there was one at this point: we obtain a final system which is also an integervalued measure. The presentation and the existence of those direct transformations are done in Section 2 - we say direct since the cloud is chosen at once from the initial condition. The absolute continuity question which we study here is to find sufficient conditions on $f$ to ensure that the distribution of the final system is absolutely continuous with respect to the distribution $\mathbb{P}$ of the initial state - this Absolute Continuity question (AC) is detailed in Section 2.2. Notice that the stochastic nature of this transformation is a great difference with the deterministic transformations of the Brownian paths.

One of the main results of Picard [10] is that a large class of such transformations, even associated with a more general cloud distribution, has the wished absolute continuity property if the cloud is almost surely finite. This result uses a stochastic calculus, developed around the finite difference gradient of Nualart and Vives [9] and Duality Formulae that we recall in Proposition A.1 of Appendix A. As a consequence, a direct transformation having finite clouds has the absolute continuity property; Section 3.1 
investigates this easy case and we state Theorem 3.1 which gives a sufficient condition on $f$ to be in this case and which solves the absolute continuity problem.

Then we turn to a more general case of direct transformation, where the cloud is allowed to be infinite. As we know how to deal with the finite case, we intersect in Section 3.2 the cloud with an increasing collection $\left(U_{t}\right)_{t}$ covering $U$ and satisfying some properties, which we call a direction. This notion of direction has been already used in [7] and was recalled in Section 1. Hence we obtain a collection of intermediate transformations indexed by $t \in \mathbb{R}^{+}$, entering in the finite framework of Theorem 3.1 and for which we know the absolute continuity property. This gives an explicit collection $\left(L_{t}\right)_{t}$ of intermediate Radon-Nikodym derivatives. Following the strategy of Lemma 3.1, we could conclude if we were able to estimate $\mathbb{E}\left[L_{t}^{2}\right]$ or $\mathbb{E}\left[L_{t} \log L_{t}\right]$ uniformly in $t$ but it is not possible because of the complex form of $L_{t}$. Thus we follow the idea of Enchev-Stroock and try to relate the direct transformation with the Poisson analogue to the (3)-type transformations, that is, the Markovian transformations introduced by Picard [11] for which we have sufficient conditions for the absolute continuity problem thanks to [7]. Proposition 3.2 shows that one can always relate in a certain sense a direct transformation with a Markovian one, thanks to a Bayes-type formula. Despite of this, we are unable to use the results of [7] in a general anticipative context, mainly because our transformations are stochastic - whereas this transfer of result was possible in the Wiener context.

However, the representation property solves the case of an adapted intensity $f$ : this allows us to turn to another problem consisting in studying the case of an adapted cloud plus a finite, anticipative cloud. The result is formalized in Theorem 3.3, where we give a sufficient condition in the case of only adding particles. We outline that, even if this main theorem is given in the case of only adding particles, we are obliged to consider in this paper direct transformations which also remove particles for the proof of the theorem.

We give then in Section 4 some applications to the anticipative Theorem 3.3 and we especially turn back to our original problem in Section 4.3. The next and last section gives the results of Picard [10] which we need in this paper and a technical estimation result.

For a measurable space $(X, \mathcal{X})$, we will denote by $\mathcal{E}_{b}(X, \mathcal{X})$, or sometimes $\mathcal{E}_{b}(X)$ when there is no ambiguity the set of bounded $\mathcal{X}$-measurable, real-valued mappings on $X$. We will also denote by $\mathcal{M}_{1}(X, \mathcal{X})$, or sometimes $\mathcal{M}_{1}(X)$ the set of probabilities on $(X, \mathcal{X})$.

\section{Poisson space}

\subsection{Definitions}

Let $(U, \mathcal{U})$ be a Lusin space, $\lambda^{-}$be a $\sigma$-finite, diffuse measure on $(U, \mathcal{U})$. We note $\Omega$ the set of integer-valued measures on $U$ such that

$-\omega(\{u\}) \leqslant 1$ for all $u$,

$-\omega(A)<\infty$ if $\lambda^{-}(A)<\infty$. 
For $V \in \mathcal{U}$ we note $\omega_{\mid V}$ the restriction of $\omega$ to $V$, identified as an element of $\Omega$. We consider the canonical random measure on $U$ defined by

$$
\lambda^{+}(\omega, A)=\omega(A)
$$

and the $\sigma$-field $\mathcal{F}$ generated by the mappings $A \mapsto \lambda^{+}(A), A \in \mathcal{U}$; note that we do not complete $\mathcal{F}$ with respect to $\mathbb{P}$. It may be seen that $(\Omega, \mathcal{F})$ is a Lusin space. We consider now the Poisson probability $\mathbb{P}$ on $(\Omega, \mathcal{F})$ such that

- for $A \in \mathcal{U}, \lambda^{+}(A)$ is a Poisson variable with parameter $\lambda^{-}(A)$,

- for $\left(A_{i}\right) \subset \mathcal{U}$ being disjoint sets, the random variables $\left(\lambda^{+}\left(A_{i}\right)\right)$ are independent.

Remark that an element $\omega$ may be seen as a particle system on $U$ whose particles are $\{u, \omega(\{u\})=1\}$. By a convenient abuse of notation we will sometimes identify a point measure to its support. We call a process on $U \times \Omega$ every measurable mapping from $(U \times \Omega, \mathcal{U} \otimes \mathcal{F})$ to $\mathbb{R}$. We denote by $\lambda$ the compensated measure on $U$ given by

$$
\lambda(\omega, d u)=\left(\lambda^{+}-\lambda^{-}\right)(\omega, d u) .
$$

We also define $|\lambda|$ by

$$
|\lambda|(\omega, d u)=\left(\lambda^{+}+\lambda^{-}\right)(\omega, d u) .
$$

For $u \in U$ we define the flip operator $\varepsilon_{u}: \Omega \rightarrow \Omega$ by $-\delta_{u}$ stands for a Dirac mass in $u$ :

$$
\varepsilon_{u}(\omega)= \begin{cases}\omega+\delta_{u} & \text { if } u \notin \omega \\ \omega-\delta_{u} & \text { else. }\end{cases}
$$

This operator acts on $\omega$ by removing the particle in $u$ if there was one or adding a particle if there was not one. For $u \in U$ and $F \in \mathcal{E}_{b}(\Omega)$ we will note

$$
\bar{D}_{u} F=F \circ \varepsilon_{u}-F .
$$

Notice that this operator has at most a sign difference with the operator $D_{u}$ used in [10, 11] and [7]. We also define the measures

$$
\mu^{ \pm}(d u, d \omega)=\lambda^{ \pm}(\omega, d u) \otimes \mathbb{P}(d \omega)
$$

on $(U \times \Omega, \mathcal{U} \otimes \mathcal{F})$ and we note

$$
\mu(d u, d \omega)=\lambda(\omega, d u) \otimes \mathbb{P}(d \omega), \quad|\mu|(d u, d \omega)=|\lambda|(\omega, d u) \otimes \mathbb{P}(d \omega) .
$$

We turn now to the notion of direction, introduced in [7]. Consider a non decreasing collection $\left(U_{t}\right)_{t \in \mathbb{R}^{+}} \subset \mathcal{U}$ of sets of $U$, such that $\bigcup_{t} U_{t}=U$. For $t>0$, we note $U_{t^{-}}=$ $\bigcup_{s<t} U_{s}$ for $t>0, U_{0^{-}}=U_{0}$ and $\delta U_{t}=U_{t} \backslash U_{t^{-}}$. We now define the gauge function as

$$
G(u)=\inf \left\{t \geqslant 0, u \in U_{t}\right\}
$$

and the growth measure of $\left(U_{t}\right)_{t}$ as the positive measure $\lambda_{(U)}^{-}$on $\left(\mathbb{R}^{+}, \mathcal{B}\left(\mathbb{R}^{+}\right)\right)$given by

$$
\lambda_{(U)}^{-}=\lambda^{-} \circ G^{-1} .
$$


Such a collection will be called a direction if it satisfies both:

$$
\text { (RC): } \bigcap_{s>t} U_{s}=U_{t} \quad \text { (Right Continuity), }
$$

(GC): $\lambda_{(U)}^{-}$is diffuse and for every $t, \lambda^{-}\left(U_{t}\right)<\infty \quad$ (Growth Condition).

Remark that $u \in \delta U_{G(u)}$ for all $u$ and that $G:(U, \mathcal{U}) \rightarrow\left(\mathbb{R}^{+}, \mathcal{B}\left(\mathbb{R}^{+}\right)\right)$is finite and measurable. Up to a change of time variable, we will assume that $\lambda_{(U)}^{-}(d t)=d t$. Remark that (GC) implies that $\lambda^{-}\left(\delta U_{t}\right)=0$ for all $t$. The second part of (GC), namely, $\lambda^{-}\left(U_{t}\right)<\infty$ is called the finite volume assumption. For sake of clearness we will denote by $U_{I}$ for $I$ being a subset of $\mathbb{R}^{+}$the set of $u$ such that $G(u) \in I$. We note $\mathcal{F}_{t}=\mathcal{F}_{U_{t}}$ and for $V \subset U$ we note $V_{t}=V \cap U_{t}$. We now give an important example. We end this part by giving the definition of an adapted/predictable process with respect to a direction. We say that a process $h$ is adapted if $h_{u}($.$) is \mathcal{F}_{G(u)}$-measurable for all $u \in U$ and that it is predictable if $h_{u}($.$) is \mathcal{F}_{G(u)^{-}}$-measurable for all $u \in U$ : we outline that both notions depend on the choice of a direction $\left(U_{t}\right)_{t}$. Those definitions are not the classical ones but the reader may satisfythat, since we do not complete $\mathcal{F}$, they coincide with the classical ones for instance in the case of the canonical example.

\subsection{The canonical example}

Suppose $U=[0 ; 1] \times\left(\mathbb{R}^{d} \backslash\{0\}\right)$ for some $d \geqslant 1$. We define $\lambda^{-}(d t, d x)=d t \mu(d x)$ where $d t$ stands for the Lebesgue measure on $[0 ; 1]$ and $\mu$ is a positive, $\sigma$-finite measure on $\mathbb{R}^{d}$ satisfying $-|$.$| denotes the Euclidean norm on \mathbb{R}^{d}$ :

$$
\int_{\mathbb{R}^{d}}\left(|x|^{2} \wedge 1\right) \mu(d x)<\infty
$$

In this setup, the process

$$
X_{t}(\omega)=\int_{[0 ; t] \times\{x:|x| \leqslant 1\}} x \lambda(\omega, d s, d x)+\int_{[0 ; t] \times\{x:|x|>1\}} x \lambda^{+}(\omega, d s, d x)
$$

is a Lévy process with Lévy measure $\mu$. The reader will easily satisfythat the collection $\left(U_{s}\right)_{s}$ given by

$$
U_{s}=[0 ; 1] \times\{x:|x| \geqslant 1 / s\}
$$

is a direction on $U$. We have in this case that $G(t, x)=1 /|x|$ and $\delta U_{s}=[0 ; 1] \times\{x:|x|=$ $1 / s\}$. Remember that we note $\mathcal{F}_{t}=\mathcal{F}_{U_{t}}$, hence $\left(\mathcal{F}_{t}\right)_{t}$ is a filtration. Notice that it differs from the classical filtration $\left(\mathcal{F}_{s}^{\prime}\right)_{s}$ usually considered in this setup, where $\mathcal{F}_{s}^{\prime}$ is defined by

$$
\mathcal{F}_{s}^{\prime}=\sigma\left\{X_{r}, r \in[0 ; s]\right\} .
$$

In our context, the past at time $t$ is considered by the events depending on the jumps of $X$. whose size is larger than $1 / t$. 


\section{Definition of a direct transformation and objective}

We give in Section 2.1 the definition and the properties of a direct transformation. The objective of the paper is given in Section 2.2, whereas the technical Section 2.3 gives a mathematical proof of the existence of such transformations. Before turning to all these points, we introduce some useful definitions.

We consider a triplet $\left(U, \mathcal{U}, \lambda^{-}\right)$as above, equipped with a direction $\left(U_{t}\right)_{t}$. For $k \in \mathbb{N}$ and $V \subset U$ we introduce $P_{k}(V)=\{W \subset V:|W|=k\}$ and $S_{k}(V)=\left\{\left(u_{1}, \ldots, u_{k}\right) \in\right.$ $V^{k}: u_{i} \neq u_{j}$ for every $\left.i \neq j\right\}$; we note $P(V)=\bigcup_{k} P_{k}(V)$ and $S(V)=\bigcup_{k} S_{k}(V)$. There is a natural projection $\pi: S(V) \rightarrow P(V)$ and we endow $P(V)$ with the smallest $\sigma$ field $\mathcal{P}(U)$ such that $\pi$ is measurable. If $m$ is a measure on $U$, we can consider $m^{\otimes^{k}}$ the product measure on $U^{k}$, with $m^{\otimes^{0}}$ defined as a Dirac mass in $\emptyset$; if we consider the restriction of $m^{\otimes^{k}}$ to $S_{k}(U)$ we can obtain a measure on $S(U)$, that we rewrite $m$, given by

$$
m(d \tau)=m\left(d u_{1}\right) \ldots m\left(d u_{k}\right)
$$

for $\tau=\left(u_{1}, \ldots, u_{k}\right)$. One can extend this measure to $P(U)-$ we will rewrite it $m-$ defined on each $P_{k}(U)$ by

$$
m(A)=m\left(\pi^{-1}(A)\right) / k !
$$

If we apply this construction to $m:=|\lambda|$ we obtain a new measure $|\lambda|$ on $P(U)$ and we consider the measure $v$ on $P(U) \times \Omega$ given by

$$
v(d A, d \omega)=|\lambda|(\omega, d A) \mathbb{P}(d \omega) .
$$

Thus we have

$$
\int Z d|\lambda|=\sum_{k} \int_{S_{k}(U)} Z_{\left\{u_{1}, \ldots, u_{k}\right\}}|\lambda|\left(d u_{1}\right) \ldots|\lambda|\left(d u_{k}\right) / k !
$$

and

$$
\int Z d v=\sum_{k} \mathbb{E}\left[\int_{S_{k}(U)} Z_{\left\{u_{1}, \ldots, u_{k}\right\}}|\lambda|\left(d u_{1}\right) \ldots|\lambda|\left(d u_{k}\right)\right] / k !
$$

for any process $Z$ on $P(U) \times \Omega$. We can as well lift the measures $\lambda^{-}$and $\lambda^{+}$up on $P(U)$. Remark that the lifted measures are $\sigma$-finite: there will be no problem to use Fubini and Lebesgue theorems. We now introduce the cloud set $\widetilde{\Omega}=\left\{V \subset U: V \cap U_{t} \in P\left(U_{t}\right), t \in\right.$ $\left.\mathbb{R}^{+}\right\}$. We endow it with the $\sigma$-field $\widetilde{\mathcal{F}}=\sigma\left\{\tilde{\omega} \mapsto \tilde{\omega}_{t}, t \in \mathbb{R}^{+}\right\}$. It may be seen that $(\widetilde{\Omega}, \widetilde{\mathcal{F}})$ is a Lusin space. For $\tilde{\omega}=\left\{u_{1}, u_{2}, \ldots\right\} \in \widetilde{\Omega}$ and $\omega \in \Omega$ we note

$$
\varepsilon_{\tilde{\omega}}(\omega)=\left(\varepsilon_{u_{1}} \circ \varepsilon_{u_{2}} \circ \cdots\right)(\omega) .
$$

By a convenient abuse of notation, we will sometimes identify an element $\tilde{\omega} \in \widetilde{\Omega}$ to a point measure on $U$, the support of which being given by $\tilde{\omega}$ whereas an element of $\Omega$ may also be seen as an element of $\widetilde{\Omega}$. Notice that the sets $\widetilde{\Omega}$ and $\Omega$ are actually similar despite 
of a different transformation. However we prefer to use different notations to design the Poisson set $\Omega$ and the set of clouds $\widetilde{\Omega}$ in order to avoid confusions. Let $f: U \times \Omega \rightarrow \mathbb{R}^{+}$ a measurable mapping satisfying

$$
(\mathrm{H}): \quad\left\{\begin{array}{l}
f \text { is bounded on each } U_{t} \times \Omega, \\
\text { For every } u \in \omega, f_{u}(\omega) \leqslant 1
\end{array}\right.
$$

\subsection{Properties of the transformation}

Let $\omega \in \Omega$. The first aim of the present work will be to build a collection of probabilities $\left(\widetilde{\mathbb{P}}_{\omega}^{f}\right)_{\omega} \subset \mathcal{M}_{1}(\widetilde{\Omega}, \widetilde{\mathcal{F}})$ such that

(A) For $V \in \mathcal{U}$ such that $\omega(V)=0, \tilde{\omega}(V)$ has a Poisson distribution with parameter $\int_{V} f_{u}(\omega) \lambda^{-}(d u)$ under $\widetilde{\mathbb{P}}_{\omega}^{f}$

(B) For $(V, W) \subset \mathcal{U}$ being disjoint, then $\tilde{\omega}(V)$ and $\tilde{\omega}(W)$ are independent under $\widetilde{\mathbb{P}}_{\omega}^{f}$,

(C) For $u \in \omega, u \in \tilde{\omega}$ with probability $f_{u}(\omega)$,

(D) $\omega \mapsto \widetilde{\mathbb{P}}_{\omega}^{f}[F]$ is measurable for every $F \in \mathcal{E}_{b}\left(\Omega_{D}\right)$.

The proof of the existence of such probabilities will be done in Section 2.3, but we first assume that we have such a collection $\left(\widetilde{\mathbb{P}}_{\omega}^{f}\right)_{\omega \in \Omega}$.

\subsection{Objective}

We note $\mathbb{P}_{\otimes}^{f} \in \mathcal{M}_{1}(\Omega \times \widetilde{\Omega}, \mathcal{F} \otimes \widetilde{\mathcal{F}})$ the probability defined by

$$
\mathbb{P}_{\otimes}^{f}(d \omega, d \tilde{\omega})=\widetilde{\mathbb{P}}_{\omega}^{f}(d \tilde{\omega}) \mathbb{P}(d \omega) .
$$

It is well defined thanks to (D). The aim of this paper is to find sufficient conditions on $f$ to have the existence of $L \in L^{1}(\Omega, \mathcal{F}, \mathbb{P})$ such that

$$
\text { (AC): For every } F \in \mathcal{E}_{b}(\Omega), \quad \mathbb{E}_{\otimes}^{f}\left[F\left(\varepsilon_{\tilde{\omega}}(\omega)\right)\right]=\mathbb{E}[F L] .
$$

This is equivalent to ask that the distribution of the final system $Y_{\infty}=\varepsilon_{\tilde{\omega}} \omega$ under $\mathbb{P}_{\otimes}^{f}$ is absolutely continuous with respect to the Poisson distribution $\mathbb{P}$ of the initial system $Y_{0}=\omega$. Notice that the final system $Y_{\infty}$ is the initial system, the particles of which being in $\omega \cap \tilde{\omega}$ have been removed, and the particles of which in $\tilde{\omega} \backslash \omega$ have been added. We end this section by proving in the following part the existence of the collection $\left(\widetilde{\mathbb{P}}_{\omega}^{f}\right)_{\omega}$.

\subsection{Existence of the transformation}

This section is devoted to the construction of the sequence $\left(\widetilde{\mathbb{P}}_{\omega}^{f}\right)_{\omega \in \Omega}$ satisfying the four above points. We formulate this construction in the following proposition

Proposition 2.1. - Let $f$ satisfying $(\mathrm{H})$ and $\omega \in \Omega$. There exists a unique probability $\widetilde{\mathbb{P}}_{\omega}^{f} \in \mathcal{M}_{1}(\widetilde{\Omega}, \widetilde{\mathcal{F}})$ such that for every $t \in \mathbb{R}^{+}$,

$$
\widetilde{\mathbb{P}}_{\omega}^{f}\left[\tilde{\omega}_{t} \in \mathcal{A}\right]=\int_{\mathcal{A}} Z_{\omega}^{f, t}(A)|\lambda|(\omega, d A) \quad \text { for every } \mathcal{A} \in \mathcal{P}(U),
$$




$$
\begin{aligned}
Z_{\omega}^{f, t}(A)= & \exp \left(\int_{U_{t} \backslash A} \log \left(1-f_{u}(\omega)\right) \lambda^{+}(\omega, d u)-\int_{U_{t}} f_{u}(\omega) \lambda^{-}(d u)\right) \\
& \times\left(\prod_{u \in A} f_{u}(\omega)\right) 1_{\left\{A \subset U_{t}\right\}} .
\end{aligned}
$$

Moreover, the given collection $\left(\widetilde{\mathbb{P}}_{\omega}^{f}\right)_{\omega \in \Omega}$ satisfies the points (A) to (D).

We also define $Z_{\omega}^{f, t^{-}}(A)$ by replacing $U_{t}$ by $U_{t^{-}}$in the expression of $Z_{\omega}^{f, t}(\tilde{\omega})$.

Proof. - Observe that for any $t \in \mathbb{R}^{+}$, and $A, B$ being disjoint subsets of $U_{t}$ we have

$$
Z_{\omega}^{f, t}(A \cup B)=\frac{Z_{\omega}^{f, t}(A) Z_{\omega}^{f, t}(B)}{K_{t}(\omega)}
$$

where

$$
K_{t}=\exp \left(\int_{U_{t}} \log \left(1-f_{u}(\omega)\right) \lambda^{+}(\omega, d u)-\int_{U_{t}} f_{u}(\omega) \lambda^{-}(d u)\right) .
$$

On the other hand, for $\Psi_{1}$ and $\Psi_{2}$ being two positive mappings on $P(U), V$ and $W$ being a partition of $U_{t}$ then one can show that

$$
\begin{aligned}
& \int_{P\left(U_{t}\right)} \Psi_{1}(A \cap V) \Psi_{2}(A \cap W)|\lambda|(\omega, d A) \\
& =\left(\int_{P(V)} \Psi_{1}(A)|\lambda|(\omega, d A)\right)\left(\int_{P(W)} \Psi_{2}(A)|\lambda|(\omega, d A)\right) .
\end{aligned}
$$

By using the same notations and (9) this gives

$$
\begin{aligned}
& \int_{P\left(U_{t}\right)} \Psi_{1}(A \cap V) \Psi_{2}(A \cap W) Z_{\omega}^{f, t}(A)|\lambda|(\omega, d A) \\
& \quad=K_{t}^{-1}\left(\int_{P(V)} \Psi_{1}(A) Z_{\omega}^{f, t}(A)|\lambda|(\omega, d A)\right)\left(\int_{P(W)} \Psi_{2}(A) Z_{\omega}^{f, t}(A)|\lambda|(\omega, d A)\right) .
\end{aligned}
$$

We now show that $Z_{\omega}^{f, t}($.$) is a probability density with respect to the measure |\lambda|$ on $P(U)$ for every $t$, that is,

$$
\int_{P(U)} Z_{\omega}^{f, t}(A)|\lambda|(\omega, d A)=1
$$

Suppose first that $f_{u}(\omega)=0$ if $u \in \omega$. In this case, we have that $Z_{\omega}^{f, t}(A)=0$ if $A \cap \omega \neq \emptyset$. This leads to

$$
\int_{P(U)} Z_{\omega}^{f, t}(A)|\lambda|(\omega, d A)=\int_{P(U)} Z_{\omega}^{f, t}(A) \lambda^{-}(d A)
$$




$$
\begin{aligned}
& =\exp \left(-\int_{U_{t}} f_{u}(\omega) \lambda^{-}(d u)\right)\left(\sum_{n \geqslant 0} \frac{1}{n !} \int_{\left(U_{t}\right)^{n}} \prod_{i=1}^{n}\left(f_{u_{i}}(\omega) \lambda^{-}\left(d u_{i}\right)\right)\right) \\
& =1 .
\end{aligned}
$$

On the other hand, suppose that $f_{u}(\omega)=0$ if $u \notin \omega$ : we obtain that $Z_{\omega}^{f, t}(A)=0$ if $A \not \subset \omega$. Since $\lambda^{-}$is assumed to be diffuse, one can see in this case that:

$$
\begin{aligned}
\int_{P(U)} Z_{\omega}^{f, t}(A)|\lambda|(\omega, d A) & =\int_{P(U)} Z_{\omega}^{f, t}(A) \lambda^{+}(\omega, d A) \\
& =\sum_{A \subset\left(\omega \cap U_{t}\right)}\left(\left(\prod_{u \in A} f_{u}(\omega)\right) \times\left(\prod_{u \in\left(\omega \cap U_{t}\right) \backslash A}\left(1-f_{u}(\omega)\right)\right)\right) \\
& =\prod_{u \in \omega \cap U_{t}}\left(1-f_{u}(\omega)+f_{u}(\omega)\right)=1
\end{aligned}
$$

and we also have (11). Consider now a process $f$ and set $f_{u}^{1}(\omega)=\omega(u) f_{u}(\omega)$ and $f_{u}^{2}(\omega)=(1-\omega(u)) f_{u}(\omega)$. By using (10) with $\Psi_{1} \equiv \Psi_{2} \equiv 1, V=U_{t} \cap \omega, W=U_{t} \backslash V$ we obtain

$$
\begin{aligned}
& \int_{P(U)} Z_{\omega}^{f, t}(A)|\lambda|(\omega, d A) \\
& \quad=K_{t}^{-1}\left(\int_{P\left(U_{t} \cap \omega\right)} Z_{\omega}^{f, t}(A)|\lambda|(\omega, d A)\right) \times\left(\int_{P\left(U_{t} \backslash \omega\right)} Z_{\omega}^{f, t}(A)|\lambda|(\omega, d A)\right) .
\end{aligned}
$$

Since $\lambda^{-}$is diffuse, we have that

$$
Z_{\omega}^{f, t}(A)=Z_{\omega}^{f^{1}, t}(A) \exp \left(-\int_{U_{t}} f_{u}(\omega) \lambda^{-}(d u)\right)
$$

for $A \subset U_{t} \cap \omega$ and

$$
Z_{\omega}^{f, t}(A)=Z_{\omega}^{f^{2}, t}(A) \exp \left(\int_{U_{t}} \log \left(1-f_{u}(\omega)\right) \lambda^{+}(\omega, d u)\right)
$$

for $A \subset U_{t} \backslash \omega$. Then we finally obtain that

$$
\begin{aligned}
& \int_{P(U)} Z_{\omega}^{f, t}(A)|\lambda|(\omega, d A) \\
& \quad=\left(\int_{P\left(U_{t}\right)} Z_{\omega}^{f^{1}, t}(A)|\lambda|(\omega, d A)\right)\left(\int_{P\left(U_{t}\right)} Z_{\omega}^{f^{2}, t}(A)|\lambda|(\omega, d A)\right)
\end{aligned}
$$

and we conclude by using both last cases applied to $f^{1}$ and $f^{2}$. We turn now to the question of the existence of $\widetilde{\mathbb{P}}_{\omega}^{f}$. This problem may be seen as a Kolmogorov extension problem: let us note $z_{t}(\tilde{\omega})=\tilde{\omega}_{t}$, where we recall that $\tilde{\omega}_{t}=\tilde{\omega} \cap U_{t}$. This process is a 
càd làg, piecewise constant process thanks to the (RC) assumption. We are looking for a probability $\widetilde{\mathbb{P}}_{\omega}^{f} \in \mathcal{M}_{1}(\widetilde{\Omega}, \widetilde{\mathcal{F}})$ such that the finite-dimensional distributions of $z$. would be defined by: for $t_{1} \leqslant \cdots \leqslant t_{n}$ and $\left(\mathcal{A}_{i}\right)_{i=1}^{n} \subset \mathcal{P}(U)$ then

$$
\widetilde{\mathbb{P}}_{\omega}^{f}\left[\left(z_{t_{1}}, \ldots, z_{t_{n}}\right) \in \mathcal{A}_{1} \times \cdots \times \mathcal{A}_{n}\right]=\int_{P(U)} Z_{\omega}^{f, t_{n}}(A) F(A)|\lambda|(\omega, d A)
$$

with

$$
F(A)=\prod_{i=1}^{n} 1_{\left\{A_{t_{i}} \in \mathcal{A}_{i}\right\}} .
$$

One easily satisfies thanks to (10) that the right-side distributions of (12) are compatible. $z$. takes its values in the Lusin space $(P(U), \mathcal{P}(U))$, which may itself be embedded in some Polish space $(\bar{P}(U), \overline{\mathcal{P}}(U))$ for which we note $(\bar{\Omega}, \overline{\mathcal{F}})$ the space of càd làg paths taking their values in $\bar{P}(U)$ - the topology on $\bar{P}(U)$ does not matter since we work with piecewise constant processes. Thus, the extension problem may be embedded in a Polish framework and we obtain by the Kolmogorov's extension theorem a probability $\overline{\mathbb{P}}_{\omega}^{f} \in \mathcal{M}_{1}(\bar{\Omega}, \overline{\mathcal{F}})$ under which the finite-dimensional distributions of $(t, \bar{\omega}) \mapsto \bar{\omega}_{t}$ are given by (12). This implies that

$$
\overline{\mathbb{P}}_{\omega}^{f}\left[\bar{\omega}_{t} \in P(U), \forall t\right]=1
$$

if we identify $P(U)$ with their image in $\bar{\Omega}$ and one concludes by setting

$$
\widetilde{\mathbb{P}}_{\omega}^{f}[.]=\overline{\mathbb{P}}_{\omega}^{f}\left[\left\{\bar{\omega}_{t} \in P(U), \forall t\right\} \cap .\right] .
$$

We now show that the given collection satisfies the claimed points (A) to (D). (D) is obvious from the definition of $\widetilde{\mathbb{P}}_{\omega}^{f}$ and we now prove (A). Let $\omega \in \Omega, V \in \mathcal{U}$ included in some $U_{t}$ such that $\omega(V)=0$ and $n \in \mathbb{N}$. By using (10) we obtain that

$$
\begin{aligned}
\widetilde{\mathbb{P}}_{\omega}^{f}[\tilde{\omega}(V)=n]= & \int_{P\left(U_{t}\right)} 1_{\{|A \cap V|=n\}} Z_{\omega}^{f, t}(A)|\lambda|(\omega, d A) \\
= & K_{t}^{-1}\left(\int_{P(V)} 1_{\{|A|=n\}} Z_{\omega}^{f, t}(A)|\lambda|(\omega, d A)\right) \\
& \times\left(\int_{P\left(U_{t} \backslash V\right)} Z_{\omega}^{f, t}(A)|\lambda|(\omega, d A)\right) .
\end{aligned}
$$

The second integral of the above product is equal to - we use that $\omega(V)=0$ :

$$
\begin{gathered}
\left(\int_{U_{t} \backslash V_{t}} \exp \left(\int_{\left(U_{t} \backslash V\right) \backslash A} \log \left(1-f_{u}(\omega)\right) \lambda^{+}(\omega, d u)-\int_{U_{t} \backslash V} f_{u}(\omega) \lambda^{-}(d u)\right)\right. \\
\left.\times\left(\prod_{u \in A} f_{u}(\omega)\right)|\lambda|(\omega, d A)\right) \exp \left(-\int_{V} f_{u}(\omega) \lambda^{-}(d u)\right)
\end{gathered}
$$




$$
=\exp \left(-\int_{V} f_{u}(\omega) \lambda^{-}(d u)\right)
$$

since one shows that the first term is equal to 1 as we proved (11). On the other hand, observe that

$$
Z_{\omega}^{f, t}(A)|\lambda|(\omega, d A)=K_{t}\left(\prod_{u \in A} f_{u}(\omega)\right) \lambda^{-}(d A)
$$

if $A \subset V$, hence the first integral of the (13) is equal to

$$
\frac{\left(\int_{V} f_{u}(\omega) \lambda^{-}(d u)\right)^{n}}{n !} .
$$

This gives (A). (C) comes from a similar computation and the independence (B) straightforward comes from (10).

We end this section by a technical integration result - remember that we sometimes identify an element $\tilde{\omega} \in \widetilde{\Omega}$ to a point measure on $U$ :

LEMMA 2.1. - Let $g: U^{n} \times \Omega \rightarrow \mathbb{R}^{+}$, vanishing if two parameters are equal. Then for every $\omega$ we have

$$
\widetilde{\mathbb{E}}_{\omega}^{f}\left[\int_{U^{n}} g_{u_{1} \ldots u_{n}}(\omega) \tilde{\omega}\left(d u_{1}\right) \cdots \tilde{\omega}\left(d u_{n}\right)\right]=\int_{U^{n}} g_{u_{1} \ldots u_{n}}(\omega) \prod_{i=1}^{n}\left(f_{u_{i}}(\omega)|\lambda|\left(\omega, d u_{i}\right)\right) .
$$

Proof. - We just show the relation in case of a simple $g$ having the form

$$
\prod_{i=1}^{n}\left(1_{V_{i}}\left(u_{i}\right)\right) F(\omega)
$$

where $\left(V_{i}\right)_{i}$ is a collection of $\lambda^{-}$-finite subsets of $U$ with $V_{i} \cap V_{j}=\emptyset$ if $i \neq j$. Observe that the property (B) implies the independence of the random variables $\tilde{\omega}\left(V_{i}\right)$ under $\widetilde{\mathbb{P}}_{\omega}^{f}$ thanks to an induction argument on $n$. Then we use the points (A) to (C) to compute the expectation and obtain the desired result.

\section{Analysis of the (AC) problem}

We turn back to the (AC) question. The first case we investigate is a rather simple situation where an additional assumption on $f$ will imply that the cloud $\widetilde{\omega}$ is almost surely finite. In this case, the (AC) question is a direct consequence of the results from Picard [10].

\subsection{The case of a finite cloud}

We suppose in this part that the following assumption is in force:

$$
\text { (L1): } \int_{U} f_{u}(\omega)|\lambda|(\omega, d u)<\infty, \quad \mathbb{P} \text { a.s. }
$$


and we note $\Omega_{0}=\left\{\omega: \int_{U} f_{u}(\omega)|\lambda|(\omega, d u)<\infty\right\}$. It may be seen from Lemma 2.1 that

$$
\widetilde{\mathbb{E}}_{\omega}^{f}[\tilde{\omega}(U)]=\int_{U} f_{u}(\omega)|\lambda|(\omega, d u)
$$

hence the (L1) assumption implies that for $\omega \in \Omega_{0}, \tilde{\omega}$ is $\widetilde{\mathbb{P}}_{\omega}^{f}$-almost surely finite. In this case, the (AC) question may be considered as a particular result of Proposition 2 of Picard [10]; this is what tells the following theorem. For any process $X(A)$ indexed by $P(U)$, we define another process $X^{\prime}(A)$ defined by $X^{\prime}(A)=X(A) \circ \varepsilon_{A}$. We will also note $Y_{\infty}: \Omega \times \widetilde{\Omega} \rightarrow \Omega$ defined by

$$
Y_{\infty}(\omega, \tilde{\omega})=\varepsilon_{\tilde{\omega}}(\omega)
$$

and we will note $Z_{\omega}^{f, \infty}($.$) the P(U)$ indexed process given in Proposition 2.1 with $t:=\infty$.

THEOREM 3.1. - Suppose that $f$ satisfies $(\mathrm{H})$ and $(\mathrm{L} 1)$. Then for $\omega \in \Omega_{0}$ we have

$$
\frac{d \widetilde{\mathbb{P}}_{\omega}^{f}}{d|\lambda|(\omega, .)}=Z_{\omega}^{f, \infty}(.)
$$

Moreover, $\mathbb{P}_{\otimes}^{f} \circ Y_{\infty}^{-1} \ll \mathbb{P}$ - that is, (AC) is in force - with

$$
\frac{d \mathbb{P}_{\otimes}^{f} \circ Y_{\infty}^{-1}}{d \mathbb{P}}=\int_{P(U)}\left(Z_{\omega}^{f, \infty}\right)^{\prime}(A)|\lambda|(\omega, d A) .
$$

Proof. - Remember that for every $t \in \mathbb{R}^{+}$and $V \subset U$ we note $V_{t}=V \cap U_{t}$. Let $\omega \in \Omega_{0}$. Then $\tilde{\omega} \in P(U), \widetilde{\mathbb{P}}_{\omega}^{f}$-almost surely; by using (A), (B) and (C) we have that, for every $\mathcal{A} \in \mathcal{P}(U)$,

$$
\begin{aligned}
\widetilde{\mathbb{P}}_{\omega}^{f}[\tilde{\omega} \in \mathcal{A}]= & \lim _{n} \widetilde{\mathbb{P}}_{\omega}^{f}\left[\tilde{\omega}_{n} \in \mathcal{A}, \tilde{\omega} \backslash U_{n}=\emptyset\right] \\
= & \lim _{n}\left(\widetilde{\mathbb{P}}_{\omega}^{f}\left[\tilde{\omega}_{n} \in \mathcal{A}\right] \widetilde{\mathbb{P}}_{\omega}^{f}\left[\tilde{\omega} \backslash U_{n}=\emptyset\right]\right) \\
= & \lim _{n}\left(\int _ { \mathcal { A } } Z _ { \omega } ^ { f , n } ( A ) | \lambda | ( \omega , d A ) \operatorname { e x p } \left(-\int_{U \backslash U_{n}} f_{u}(\omega) \lambda^{-}(d u)\right.\right. \\
& \left.\left.+\int_{U \backslash U_{n}} \log \left(1-f_{u}(\omega)\right) \omega(d u)\right)\right)
\end{aligned}
$$

the exponential term tends to 1 as $n \rightarrow \infty$ since $\omega \in \Omega_{0}$ and by a Lebesgue dominated convergence theorem we can show that the first integral tends to $\int_{\mathcal{A}} Z_{\omega}^{f, \infty}(A)|\lambda|(\omega, d A)$. It remains to prove (AC). Let $F \in \mathcal{E}_{b}(\Omega)$. We have by using the multiple Duality Formula - see Proposition A.1 in Appendix A - and what we proved above that

$$
\mathbb{E}_{\otimes}^{f}\left[F\left(Y_{\infty}\right)\right]=\int_{P(U) \times \Omega} F\left(\varepsilon_{A} \omega\right) Z_{\omega}^{f, \infty}(A) v(d \omega, d A)
$$




$$
\begin{aligned}
& =\int_{P(U) \times \Omega} F(\omega)\left(Z_{\omega}^{f, \infty}\right)^{\prime}(A) v(d \omega, d A) \\
& =\mathbb{E}\left[F(\omega)\left(\int_{P(U)}\left(Z_{\omega}^{f, \infty}\right)^{\prime}(A)|\lambda|(d \omega, d A)\right)\right] .
\end{aligned}
$$

This concludes.

\subsection{Absolute continuity for the stopped transformations}

In the general case of our framework, where we have only $(\mathrm{H})$, the cloud $\tilde{\omega}$ is allowed to be almost surely infinite and then has no longer density with respect to $|\lambda|$. Let give us a direction $\left(U_{t}\right)_{t}$ and fix $t \in \mathbb{R}^{+}$. The assumption (H) and the definition (8) imply that the stopped cloud $\tilde{\omega}_{t}=\tilde{\omega} \cap U_{t}$ is $\widetilde{\mathbb{P}}_{\omega}^{f}$-almost surely finite: the idea is, in a first step, to apply the above results to the stopped transformations given by:

$$
Y_{t}(\omega, \tilde{\omega})=\varepsilon_{\tilde{\omega}_{t}}(\omega)
$$

Proposition 3.1. - For every $t \in \mathbb{R}^{+}, \mathbb{P}_{\otimes}^{f} \circ Y_{t}^{-1} \ll \mathbb{P}, \mathbb{P}_{\otimes}^{f} \circ Y_{t^{-}}^{-1} \ll \mathbb{P}$ with respective densities

$$
L_{t}=\int_{P(U)}\left(Z_{\omega}^{f, t}\right)^{\prime}(A)|\lambda|(\omega, d A), \quad L_{t}^{-}=\int_{P(U)}\left(Z_{\omega}^{f, t^{-}}\right)^{\prime}(A)|\lambda|(\omega, d A) .
$$

Moreover, $L$. is càd làg and $L_{t}^{-}=L_{t^{-}}$.

Notice that the collection of direct transformations $\left(Y_{t}\right)_{t}$ enters in the general framework of Section 2.2 of [7].

Proof. - The existence and the expression of $L_{t}$ may be seen as a result of the last lemma used with $f_{u}:=f_{u} 1_{U_{t}}(u)$ which satisfies (L1) for every $t$. The expression of $L_{t}^{-}$ comes from the same theorem used with $f_{u}:=f_{u} 1_{U_{t^{-}}}(u)$. The question of continuity may be solved thanks to the expressions of $L_{t}$ and $L_{t}^{-}$and dominated convergence theorems.

The problem is now to link the last result with the (AC) question. The following lemma gives one way to conclude - remember that we note $\mathcal{F}_{t}$ for $\mathcal{F}_{U_{t}}$.

LEMMA 3.1. - Let us note $\bar{L}_{t}=\mathbb{E}\left[L_{t} \mid \mathcal{F}_{t}\right]$. Then $\left\{\bar{L}_{t}, t \in \mathbb{R}^{+}\right\}$is a $\left(\mathcal{F}_{t}\right)_{t}$-martingale. Moreover, we have (AC) iff $\left\{\bar{L}_{t}, t \in \mathbb{R}^{+}\right\}$is uniformly integrable.

The lemma and its proof are similar to the basic Lemma 2.2 in [7]. Following this strategy, we could for instance try to estimate $\mathbb{E}\left[L_{t}^{2}\right]$ or $\mathbb{E}\left[L_{t} \log L_{t}\right]$ uniformly in $t$ in order to conclude for the (AC) question. But the expression given by Proposition 3.1 is too much complex and we are actually unable to estimate both last expressions. Hence we turn to another approach inspired by Enchev and Stroock [4]. 


\subsection{Markovian representation}

In Gyongy [5], the following question is investigated: let $\xi$ be a Ito process. Is it possible to find a process $X$ satisfying a simpler SDE, the one-dimensional distributions of which being the same as the ones of $\xi$ ? The answer depends on further assumptions, but the method - the use of conditional expectations - is very close to the one we will use. In Enchev and Stroock [4], a similar question concerning the study of transformations on the Wiener space is asked: let a process $f:[0 ; 1] \times \Omega \rightarrow \mathbb{R}$ be given and let $\bar{B}$ the process given by (1). Is it possible to find a process $h:[0 ; 1] \times \Omega \rightarrow \mathbb{R}$ such that the transformation (3) associated with $h$ satisfies:

$$
\mathbb{P}_{W} \circ[\tilde{B}(1, .)]^{-1}=\mathbb{P}_{W} \circ(\bar{B} .)^{-1} .
$$

In this case, the absolute continuity questions (2) and (4) concerning both transformations are equivalent: this is interesting because they know how to deal with for second one - up to regularity assumptions on $h$.

In both cases, the authors aim at representing, or mimicking some characteristics of complex systems by the ones of better known systems: we will call this a representation property. The problem we discuss here is the Poisson analogue of the EnchevStroock problem in the Wiener case. We first give the transformation which is the Poisson analogue of the (3)-type transformation. This transformation, the Markovian transformation, is defined in Section 3.3.1 and was originally introduced by Picard [11] and the related (AC)-type problem was studied in [7]. We will show in Section 3.3.2 that we have a similar representation property for the direct transformations by a Markovian one in the Poisson case, without restrictions on $f$. On the other hand, we will not be able to use the results concerning the Markovian transformations unless we are in the adapted case - that is, the case of an adapted $f$ with respect to the direction $\left(U_{t}\right)_{t}$. Despite of its lack of generality, the related Theorem 3.2 is a first success for two reasons: the first one is that we do not have a priori an easier method to deal with the adapted case. The second one is that the adapted Theorem 3.2 obtained by the Markovian representation is essential in obtaining the anticipative Theorem 3.3.

Before turning to the definition of a Markovian transformation, we give two technical lemmas. We introduce the subset $\Omega_{1}$ of $\Omega$ given by

$$
\Omega_{1}=\left\{\omega: \omega\left(\delta U_{t}\right) \leqslant 1 \text { for every } t \in \mathbb{R}^{+}\right\} .
$$

It is shown in [7] that $\mathbb{P}\left[\Omega_{1}\right]=1$ - see Proposition 2.1. We now state - remember that we note for $\bar{D}_{u} F=F \circ \varepsilon_{u}-F$ for $u \in U$ and $F \in \mathcal{E}_{b}(\Omega)$ :

LemmA 3.2. - Let $\omega \in \Omega_{1}$. Then for all $F \in \mathcal{E}_{b}(\Omega)$ and $t \in \mathbb{R}^{+}$we have

$$
\widetilde{\mathbb{E}}_{\omega}^{f}\left[F\left(Y_{t}\right)\right]=F(\omega)+\widetilde{\mathbb{E}}_{\omega}^{f}\left[\int_{U_{t}} \bar{D}_{u} F\left(Y_{G(u)^{-}}\right) f_{u}(\omega)|\lambda|(\omega, d u)\right] .
$$

Proof. - Remember that we assume that the growth measure given by (5) on $\mathbb{R}^{+}$is the Lebesgue measure. Let $\omega \in \Omega_{1}$ and $F$ having the form $F(\omega)=\Phi(\omega(V))$ with 
$\Phi \in \mathcal{E}_{b}\left(\mathbb{R}^{+}\right)$and $V \in \mathcal{U}$. It is easy to show that, for every $a<b$ with $\omega\left(U_{] a ; b]}\right)=0$, we have by using the point (A) given in Section 2.1 that

$$
\begin{aligned}
\widetilde{\mathbb{E}}_{\omega}^{f}\left[F\left(Y_{b}\right)\right]= & \widetilde{\mathbb{E}}_{\omega}^{f}\left[\Phi\left(Y_{a}(V)+\tilde{\omega}_{\mid U_{\mid l a ; b]}}(V)\right)\right] \\
= & \widetilde{\mathbb{E}}_{\omega}^{f}\left[\Phi\left(Y_{a}(V)\right)\left(1-\int_{V \cap U_{[a ; b]}} f_{u}(\omega) \lambda^{-}(d u)\right)\right]+\mathrm{o}(b-a) \\
& +\widetilde{\mathbb{E}}_{\omega}^{f}\left[\Phi\left(Y_{a}(V)+1\right)\left(\int_{V \cap U_{[a ; b]}} f_{u}(\omega) \lambda^{-}(d u)\right)\right] \\
= & \widetilde{\mathbb{E}}_{\omega}^{f}\left[\int_{V \cap U_{[a ; b]}}\left(\Phi\left(Y_{a}(V)+1\right)-\Phi\left(Y_{a}(V)\right) f_{u}(\omega) \lambda^{-}(d u)\right]\right. \\
& +\widetilde{\mathbb{E}}_{\omega}^{f}\left[F\left(Y_{a}\right)\right]+\mathrm{o}(b-a) .
\end{aligned}
$$

Now observe that

$$
\left(\Phi\left(\omega^{\prime}(V)+1\right)-\Phi\left(\omega^{\prime}(V)\right)\right) 1_{\{u \in V\}}=\bar{D}_{u} F\left(\omega^{\prime}\right)
$$

for any $\left(u, \omega^{\prime}\right)$ such that $\omega^{\prime}(\{u\})=0$. Then

$$
\widetilde{\mathbb{E}}_{\omega}^{f}\left[F\left(Y_{b}\right)\right]=\widetilde{\mathbb{E}}_{\omega}^{f}\left[F\left(Y_{a}\right)\right]+\widetilde{\mathbb{E}}_{\omega}^{f}\left[\int_{U_{[a ; b]}} \bar{D}_{u} F\left(Y_{a}\right) f_{u}(\omega) \lambda^{-}(d u)\right]+\mathrm{o}(b-a) .
$$

Now we use that

$$
\widetilde{\mathbb{P}}_{\omega}^{f}\left[Y_{a} \neq Y_{G(u)^{-}}\right] \leqslant M(b-a)
$$

for every $u \in U_{[a ; b]}, M$ being a bound of $f$ on $U_{[a ; b]}$. We finally obtain that

$$
\widetilde{\mathbb{E}}_{\omega}^{f}\left[F\left(Y_{b}\right)\right]=\widetilde{\mathbb{E}}_{\omega}^{f}\left[\int_{U_{[a ; b]}} \bar{D}_{u} F\left(Y_{G(u)^{-}}\right) f_{u}(\omega) \lambda^{-}(d u)\right]+\widetilde{\mathbb{E}}_{\omega}^{f}\left[F\left(Y_{a}\right)\right]+\mathrm{o}(b-a) .
$$

On the other hand, for $a$ such that $\omega\left(\delta U_{a}\right)=1$, then it is clear from (C) that

$$
\widetilde{\mathbb{E}}_{\omega}^{f}\left[F\left(Y_{a}\right)\right]=\widetilde{\mathbb{E}}_{\omega}^{f}\left[F\left(Y_{a^{-}}\right)\right]+\widetilde{\mathbb{E}}_{\omega}^{f}\left[\int_{\delta U_{a}} \bar{D}_{u} F\left(Y_{G(u)^{-}}\right) f_{u}(\omega) \lambda^{+}(\omega, d u)\right] .
$$

The claimed result for such $F$ may be deduced from the last two equalities by taking subdivisions of the interval $[0 ; t]$. By using the same ideas and the independence property (B), we could as well show the result for $F$ having the form

$$
F(\omega)=\Phi\left(\omega\left(V_{1}\right), \ldots, \omega\left(V_{n}\right)\right),
$$

for disjoint subsets $\left(V_{i}\right)_{i}$; by using $(\mathrm{H}),(\mathrm{A})$ and $(\mathrm{B})$ we have for every $\left.] a ; b\right]$ such that $\omega\left(U_{[a ; b]}\right)=0$ : 


$$
\begin{aligned}
\widetilde{\mathbb{E}}_{\omega}^{f}\left[F\left(Y_{b}\right)\right]= & \widetilde{\mathbb{E}}_{\omega}^{f}\left[\left(\Phi\left(Y_{a}\left(V_{1}\right)+1, Y_{a}\left(V_{2}\right), \ldots, Y_{a}\left(V_{n}\right)\right)\right.\right. \\
& \left.\left.-F\left(Y_{a}\right)\right)\left(\int_{V_{1} \cap U_{[a ; b]}} f_{u}(\omega) \lambda^{-}(d u)\right)\right]+\cdots \\
& +\widetilde{\mathbb{E}}_{\omega}^{f}\left[\left(\Phi\left(Y_{a}\left(V_{1}\right), Y_{a}\left(V_{2}\right), \ldots, Y_{a}\left(V_{n}\right)+1\right)-F\left(Y_{a}\right)\right)\right. \\
& \left.\times\left(\int_{V_{n} \cap U_{[a ; b[}} f_{u}(\omega) \lambda^{-}(d u)\right)\right]+\mathrm{o}(b-a)+\widetilde{\mathbb{E}}_{\omega}^{f}\left[F\left(Y_{a}\right)\right],
\end{aligned}
$$

since the probability that $\tilde{\omega}$ has a particle in more than one set $V_{i} \cap U_{[a ; b[}$ is a o $(b-a)$. From this equality, one concludes as above for functions (15) and the general case comes from a monotone class argument.

LEMMA 3.3. - For every bounded process $g$ on $U \times \Omega$ and $t \in \mathbb{R}^{+}$we have

$$
\mathbb{E}_{\otimes}^{f}\left[\int_{U_{t}} g_{u}\left(Y_{G(u)^{-}}\right) \lambda^{+}(\omega, d u)\right]=\mathbb{E}\left[\int_{U_{t}} L_{G(u)^{-}} g_{u} \lambda^{+}(\omega, d u)\right] .
$$

Proof. - We can prove the relation for $g$ smaller than one and having the form

$$
g_{u}(\omega)=1_{V}(u) F(\omega)
$$

where $V \in \mathcal{U}$ and $F \in \mathcal{E}_{b}(\Omega)$. The general results then comes from a monotone class argument. Let $\left(a_{k}^{N}\right)_{k=0}^{N}$ be a subdivision of $[0 ; t]$. We have that

$$
\begin{aligned}
\mathbb{E}_{\otimes}^{f}\left[\int_{U_{t}} g_{u}\left(Y_{G(u)^{-}}\right) \lambda^{+}(\omega, d u)\right] \\
=\sum_{k=0}^{N-1} \mathbb{E}_{\otimes}^{f}\left[\int_{\left.U_{\left.1 a_{k}^{N} ; a_{k+1}^{N}\right]}\left(g_{u}\left(Y_{G(u)^{-}}\right)-g_{u}\left(Y_{a_{k}^{N}}\right)\right) \lambda^{+}(\omega, d u)\right]}\right. \\
\quad+\sum_{k=0}^{N-1} \mathbb{E}_{\otimes}^{f}\left[F\left(Y_{a_{k}^{N}}\right) \omega\left(U_{] a_{k}^{N} ; a_{k+1}^{N}\right]} \cap V\right)\right] .
\end{aligned}
$$

On the one hand we have for any $u \in U_{\left.] a_{k}^{N} ; a_{k+1}^{N}\right]}$ and $\omega$ that

$$
\widetilde{\mathbb{P}}_{\omega}^{f}\left[Y_{G(u)^{-}} \neq Y_{a_{k}^{N}}\right] \leqslant M|\lambda|\left(\omega, U_{] a_{k}^{N} ; G(u)[}\right),
$$

where $M$ is a bound of $f$ on $U_{t} \times \Omega$. Hence the first sum of (16) is bounded by

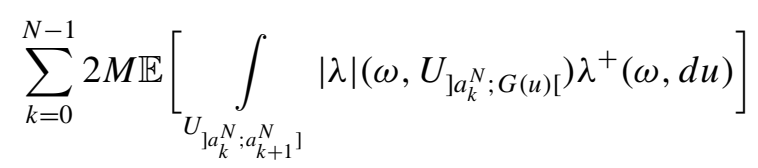


and it may be seen by a classical argument each term of the sum is a $\mathrm{o}\left(a_{k+1}^{N}-a_{k}^{N}\right)$. Hence this first sum of (16) goes to 0 as $N \rightarrow \infty$. On the other hand, by using that $\omega\left(U_{\left.] a_{k}^{N} ; a_{k+1}^{N}\right]}\right)=Y_{a_{k}^{N}}\left(U_{\left.] a_{k}^{N} ; a_{k+1}^{N}\right]}\right), \widetilde{\mathbb{P}}_{\omega}^{f}$-almost surely, one finds that the second sum of (16) is equal to

$$
\begin{aligned}
& \sum_{k=0}^{N-1} \mathbb{E}_{\otimes}^{f}\left[F\left(Y_{a_{k}^{N}}\right) \times Y_{a_{k}^{N}}\left(U_{] a_{k}^{N} ; a_{k+1}^{N}\right]} \cap V\right)\right] \\
& =\sum_{k=0}^{N-1} \mathbb{E}_{\otimes}^{f}\left[\left(F \times \omega\left(U_{] a_{k}^{N} ; a_{k+1}^{N}\right]} \cap V\right)\right) L_{a_{k}^{N}}\right]
\end{aligned}
$$

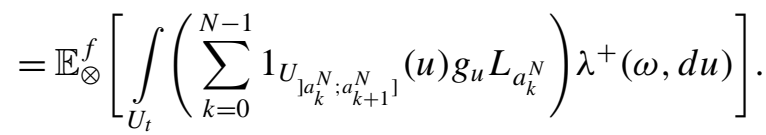

We can now easily show that this Riemann sum goes to the claimed right-side as $N \rightarrow \infty$ thanks to a dominated convergence argument based upon the bound

$$
L_{s}(\omega) \leqslant \exp \left(2 M|\lambda|\left(\omega, U_{t}\right)\right)
$$

for every $s \in[0 ; t]-$ this bound is obvious from Proposition 3.1.

\subsubsection{Definition of a Markovian transformation}

We now quickly recall what a Markovian transformation is. Let $h$ be a process satisfying $(\mathrm{H})$ and $\omega \in \Omega_{1}$. We consider on an auxiliary space $\left(\Omega^{\prime}, \mathcal{F}^{\prime}\right)$ a non homogeneous Markov process $\Lambda: \mathbb{R}^{+} \times \Omega^{\prime} \rightarrow \Omega$, càd làg, the transition probabilities being given as follows: for $[t ; t+\Delta t] \subset \mathbb{R}^{+}$such that $\omega\left(U_{] t ; t+\Delta t}\right)=0$ then

$$
\begin{gathered}
\mathbb{P}^{\prime}\left[\Lambda_{t+\Delta t}=\omega \mid \Lambda_{t}=\omega\right]=1-\int_{U_{l t ; t+\Delta t]}} h_{u}(\omega) \lambda^{-}(d u)+o(\Delta t), \\
\mathbb{P}^{\prime}\left[\Lambda_{t+\Delta t} \in\left\{\varepsilon_{u} \omega, u \in V \cap U_{l t ; t+\Delta t]}\right\} \mid \Lambda_{t}=\omega\right]=\int_{V \cap U_{l t ; t+\Delta t]}} h_{u}(\omega) \lambda^{-}(d u)+\mathrm{o}(\Delta t)
\end{gathered}
$$

for $V \in \mathcal{U}$ and if $\omega\left(\delta U_{t}\right)=1$, by noting $u$ the unique particle in $\delta U_{t}$ - remember that $\omega \in \Omega_{1}-$ then

$$
\begin{gathered}
\mathbb{P}^{\prime}\left[\Lambda_{t}=\omega \mid \Lambda_{t^{-}}=\omega\right]=1-h_{u}(\omega), \\
\mathbb{P}^{\prime}\left[\Lambda_{t}=\varepsilon_{u} \omega \mid \Lambda_{t^{-}}=\omega\right]=h_{u}(\omega) .
\end{gathered}
$$

Thus, starting from an initial condition $\Lambda_{0}=\omega \in \Omega_{1}$, we add at time $t$ such that $\omega\left(\delta U_{t}\right)=0$ a particle in $u \in \delta U_{t}$ with intensity $h_{u}\left(\Lambda_{t^{-}}\right)$with respect to $\lambda^{-}$. On the other hand, a particle of $\omega$ in $u \in \delta U_{t}$ is removed at time $t$ with probability $h_{u}\left(\Lambda_{t^{-}}\right)$. Since $\mathbb{P}\left[\Omega_{1}\right]=1$, we can define such a Markov process having $\mathbb{P}$ for initial distribution and we note $\mathbb{P}^{\prime}$ its distribution. In terms of martingale problem, this means that for any $g \in \mathcal{E}_{b}(\Omega)$

$$
\left(\mathrm{PM}^{\prime}\right): \quad M_{t}^{g}=g\left(\Lambda_{t}\right)-\int_{U_{t}}\left(\left(g \circ \varepsilon_{u}\right) h_{u}\right)\left(\Lambda_{G(u)^{-}}\right)|\lambda|\left(\Lambda_{0}, d u\right)
$$


is a martingale under the probabilities $\mathbb{P}^{\prime}\left[. \mid \Lambda_{0}=\omega\right]$. It is shown in Picard [11] that, if we note

$$
\mathbb{P}_{t}(d \omega)=\int \mathbb{P}^{\prime}\left[\Lambda_{t} \in d \omega \mid \Lambda_{0}=\omega_{0}\right] \mathbb{P}\left(d \omega_{0}\right)
$$

then $\mathbb{P}_{t} \ll \mathbb{P}$, the corresponding density $L_{t}$ being a càd làg solution to the Fokker-Planck equation:

$$
L_{t}(\omega)=1+\int_{U_{t}} \bar{D}_{u}\left[h_{u} L_{G(u)^{-}}\right]|\lambda|(\omega, d u) .
$$

It is also shown that this equation has an unique càd làg solution if $h$ satisfies $(\mathrm{H})$.

\subsubsection{Representation property}

We ask here the following question: for $f$ being given, satisfying $(\mathrm{H})$, is it possible to find some process $h$ satisfying $(\mathrm{H})$ such that for every $t \in \mathbb{R}^{+}$, the distribution of the stopped Markovian transformation $\Lambda_{t}$ is the same as the one of $Y_{t}$ under $\mathbb{P}_{\otimes}^{f}$ ? The following proposition shows that finding such a Markovian representation is always possible by a Bayes-type formula. We introduce the additional assumption

$$
(\mathrm{HM}): \quad(u, \omega) \mapsto\left(1-\omega(\{u\}) f_{u}(\omega)\right)^{-1} \text { is bounded on each } U_{t} \times \Omega .
$$

Proposition 3.2 (Markovian representation). - Assume that $f$ satisfies $(\mathrm{H})$ and (HM). Let $h: U \times \Omega \rightarrow \mathbb{R}$ be given by

$$
h_{u}(\omega)=\frac{1}{L_{G(u)^{-}}(\omega)} \int_{P(U)} f_{u}\left(\varepsilon_{A} \omega\right)\left(Z_{\omega}^{f, G(u)^{-}}\right)^{\prime}(A)|\lambda|(\omega, d A),
$$

then $h$ is measurable, satisfies $(\mathrm{H}),(\mathrm{HM})$ and we have

$$
h_{u}\left(Y_{G(u)^{-}}\right)=\mathbb{E}_{\otimes}^{f}\left[f_{u}(\omega) \mid Y_{G(u)^{-}}\right] .
$$

Let us consider the Markovian transformation $\Lambda$ defined on $\left(\Omega^{\prime}, \mathcal{F}^{\prime}, \mathbb{P}^{\prime}\right)$, associated with $h$. Then

$$
\forall t, \forall F \in \mathcal{E}_{b}(\Omega), \quad \mathbb{E}^{\prime}\left[F\left(\Lambda_{t}\right)\right]=\mathbb{E}_{\otimes}^{f}\left[F\left(Y_{t}\right)\right] .
$$

Proof. - Notice that the additional assumption (HM) implies that $L_{t^{-}}(\omega)>0$ for every $t, \omega$, then the given $h$ is well defined. The fact that $h$ fulfills (H) and (HM) may be seen from its expression. The measurability of the given process is not obvious, but may be proved by using monotone class arguments. We now show (19). Let $G \in \mathcal{E}_{b}(\Omega)$ and $u \in U$. We have

$$
\begin{aligned}
\mathbb{E}_{\otimes}^{f}\left[f_{u}(\omega) G\left(Y_{G(u)^{-}}\right)\right] & =\mathbb{E}\left[f_{u} \int_{P(U)} G\left(\varepsilon_{A} \omega\right) Z_{\omega}^{f, G(u)^{-}}(A)|\lambda|(\omega, d A)\right] \\
& =\mathbb{E}\left[\left(\int_{P(U)} f_{u}\left(\varepsilon_{A} \omega\right)\left(Z_{\omega}^{f, G(u)^{-}}\right)^{\prime}(A)|\lambda|(\omega, d A)\right) G(\omega)\right]
\end{aligned}
$$




$$
\begin{aligned}
& =\mathbb{E}\left[L_{G(u)^{-}} h_{u} G\right] \\
& =\mathbb{E}_{\otimes}^{f}\left[h_{u}\left(Y_{G(u)^{-}}\right) G\left(Y_{G(u)^{-}}\right)\right]
\end{aligned}
$$

where we have used the multiple Duality Formula of Proposition A.1 for the second equality, jointly with the fact that $\varepsilon_{A} \circ \varepsilon_{A}=$ Id. This proves (19). Now we prove (20). Consider now $F \in \mathcal{E}_{b}(\Omega)$. We obtain from Lemma 3.3 and from the given expression of $h$ that

$$
\begin{aligned}
\mathbb{E}_{\otimes}^{f} & {\left[\int_{U_{t}} \bar{D}_{u} F\left(Y_{G(u)^{-}}\right) h_{u}\left(Y_{G(u)^{-}}\right)|\lambda|(\omega, d u)\right] } \\
& =\mathbb{E}\left[\int_{U_{t}} \bar{D}_{u} F h_{u} L_{G(u)^{-}}|\lambda|(\omega, d u)\right] \\
& =\mathbb{E}\left[\int_{U} \bar{D}_{u} F\left(\int_{P(U)} f_{u}\left(\varepsilon_{A} \omega\right)\left(Z_{\omega}^{f, G(u)^{-}}\right)^{\prime}|\lambda|(\omega, d A)\right)|\lambda|(\omega, d u)\right] \\
& =\mathbb{E}\left[\int_{U_{t}}\left(\int_{P(U)} \bar{D}_{u} F\left(\varepsilon_{A} \omega\right) Z_{\omega}^{f, G(u)^{-}}(A)|\lambda|(\omega, d A)\right) f_{u}(\omega)|\lambda|(\omega, d u)\right] \\
& =\mathbb{E}_{\otimes}^{f}\left[\int_{U_{t}} \bar{D}_{u} F\left(Y_{G(u)^{-}}\right) f_{u}(\omega)|\lambda|(\omega, d u)\right]
\end{aligned}
$$

by using again the multiple Duality Formula of Proposition A.1 for the third equality. Hence, by using Lemmas 3.2 and 3.3, we obtain that for every $F \in \mathcal{E}_{b}(\Omega)$,

$$
\begin{array}{r}
\mathbb{E}_{\otimes}^{f}\left[F\left(Y_{t}\right)\right]=\mathbb{E}[F]+\mathbb{E}_{\otimes}^{f}\left[\int_{U_{t}}\left(\bar{D}_{u} F h_{u}\right)\left(Y_{G(u)^{-}}\right)|\lambda|(\omega, d u)\right] \\
\Rightarrow \quad \mathbb{E}\left[F L_{t}\right]=\mathbb{E}[F]+\mathbb{E}\left[\int_{U_{t}} \bar{D}_{u} F\left(h_{u} L_{G(u)^{-}}\right)|\lambda|(\omega, d u)\right] \\
\Rightarrow \quad \mathbb{E}\left[F L_{t}\right]=\mathbb{E}[F]+\mathbb{E}\left[F \int_{U_{t}} \bar{D}_{u}\left[h_{u} L_{G(u)^{-}}\right]|\lambda|(\omega, d u)\right]
\end{array}
$$

by using the single Duality Formula for the last line. We can deduce from this that $\left(L_{t}\right)_{t}$ satisfies the Fokker-Planck equation (18) associated with $h$. As we told above, this equation has an unique solution and this concludes the proof.

Notice that it is obvious from our definitions that $h$ is adapted (respectively predictable) if $f$ is so. This possibility of having a Markovian representation for any $f$ looks like powerful and we tried to use the results of [7] concerning the (AC) question for Markovian transformation. Unfortunately, the main result of this paper needs assumptions on the Malliavin kernel $D h$, and we are unable to relate it with the one of $D f$. However, the Markovian representation is useful in the case of an adapted $f$. 
THEOREM 3.2 (Adapted case for direct transformations). - Suppose that $f$ is an adapted process satisfying $(\mathrm{H}),(\mathrm{HM})$ and

$$
\text { (L2): } \int_{U}\left(\bar{D}_{u} f_{u}\right)^{2}(\omega) \lambda^{-}(d u)<\infty, \quad \mathbb{P} \text {-a.s. }
$$

Then we have (AC).

Proof. - We first show (AC) in the case of

$$
\mathbb{E}\left[\int_{U}\left(\bar{D}_{u} f_{u}\right)^{2} \lambda^{-}(d u)\right]<\infty .
$$

We recall now the definition of the finite difference gradient $D$ taken from Picard [10]. We introduce the operators $\varepsilon_{u}^{ \pm}$on $\Omega$ defined by

$$
\forall V \in \mathcal{U}, \quad\left(\varepsilon_{u}^{-}(\omega)\right)(V)=\omega(V \backslash\{u\}), \quad\left(\varepsilon_{u}^{+}(\omega)\right)(V)=\left(\varepsilon_{u}^{-}(\omega)\right)(V)+1_{V}(u) .
$$

Those operators are used in [10,11,7]; they give the finite difference gradient $D$ introduced by Picard [10], given by

$$
D_{u} F=F \circ \varepsilon_{u}^{+}-F \circ \varepsilon_{u}^{-}
$$

for every $u \in U$ and $F \in \mathcal{E}_{b}(\Omega)$. Notice that for every $F, u$ and $\omega$ we have

$$
\left|D_{u} F(\omega)\right|=\left|\bar{D}_{u} F(\omega)\right| .
$$

Remark now that $\bar{D}_{u} L_{G(u)^{-}}(\omega)=0$ and $\bar{D}_{u} Z_{\omega}^{f, G(u)^{-}}(A)=0$ for every $A$ since $f$ is adapted. We obtain then from the definition of $h$ that

$$
h_{u} \circ \varepsilon_{u}(\omega)=\frac{1}{L_{G(u)^{-}}(\omega)} \int_{P(U)} f_{u} \circ \varepsilon_{u}\left(\varepsilon_{A} \omega\right)\left(Z_{A}^{f, G(u)^{-}}\right)^{\prime}(\omega)|\lambda|(\omega, d A),
$$

since $\varepsilon_{u} \circ \varepsilon_{A}=\varepsilon_{A} \circ \varepsilon_{u}$ for every $A \in U_{G(u)^{-}}$. We could show from this relation that

$$
\left(\bar{D}_{u} h_{u}\right)\left(Y_{G(u)^{-}}\right)=\mathbb{E}_{\otimes}^{f}\left[\bar{D}_{u} f_{u}(\omega) \mid Y_{G(u)^{-}}\right]
$$

as we showed (19). On the other hand, one can see from the Fokker-Planck equation (18) that $L_{t}$ satisfies

$$
L_{t}=1-\int_{U_{t}} L_{G(u)^{-}}\left(D_{u} h_{u}\right) \lambda(\omega, d u),
$$

that is, $L_{t}$ is the stochastic exponential of the locally square-integrable martingale

$$
X_{t}(\omega)=-\int_{U_{t}} D_{u} h_{u} \lambda(\omega, d u)
$$


This may also be seen as a consequence of the classical Girsanov theory - see for instance Jacod and Shyryaev [6]. Notice that the process $D_{u} h_{u}$ is a predictable process, whereas the process $\bar{D}_{u} h_{u}$ is only adapted. By using an estimation of [7] concerning the Markovian transformations - see Theorem 3.2 and its proof - and (22), we obtain that

$$
\begin{aligned}
\mathbb{E}\left[L_{t} \log L_{t}\right] & \leqslant \int_{U} \mathbb{E}^{\prime}\left[\left(D_{u} h_{u}\right)^{2}\left(\Lambda_{G(u)^{-}}\right)\right] \lambda^{-}(d u) \\
& =\int_{U} \mathbb{E}^{\prime}\left[\left(\bar{D}_{u} h_{u}\right)^{2}\left(\Lambda_{G(u)^{-}}\right)\right] \lambda^{-}(d u) .
\end{aligned}
$$

By using (23) jointly with the Jensen inequality, we obtain that

$$
\sup _{t} \mathbb{E}\left[L_{t} \log L_{t}\right] \leqslant \mathbb{E}\left[\int_{U}\left(\bar{D}_{u} f_{u}\right)^{2} \lambda^{-}(d u)\right]<\infty .
$$

This concludes for the (AC) question in the case of (21), thanks to Lemma 3.1. We now turn to the general case (L2) and introduce for $k \in \mathbb{N}$ :

$$
\tau_{k}=\inf \left\{t \in \mathbb{R}^{+}: \int_{U_{t}}\left(\bar{D}_{u} f_{u}\right)^{2} \lambda^{-}(d u) \geqslant k\right\} .
$$

We consider $f_{u}^{k}(\omega)=f_{u}(\omega) \times 1_{\left\{G(u) \leqslant \tau_{k}\right\}}$. Then $f^{k}$ is an adapted process satisfying (21), (H) and (HM). On the other hand, if we note $\tilde{\omega}^{k}(\omega)=\tilde{\omega} \cap U_{\tau_{k}(\omega)}$ and

$$
Y_{t}^{k}(\omega, \tilde{\omega})=\varepsilon_{\left(\tilde{\omega}^{k}(\omega)\right)_{t}}(\omega),
$$

one easily shows that $\mathbb{P}_{\otimes}^{f} \circ\left(Y_{t}^{k}\right)^{-1}=\mathbb{P}_{\otimes}^{f^{k}} \circ Y_{t}^{-1}$. Consider now $B \in \mathcal{F}$ such that $\mathbb{P}[B]=0$. The above case used with $f:=f^{k}$ implies that

$$
\mathbb{P}_{\otimes}^{f^{k}}\left[Y_{\infty} \in B\right]=0
$$

for every $k$, then we obtain that

$$
\begin{aligned}
\mathbb{P}_{\otimes}^{f}\left[Y_{\infty} \in B\right] & =\mathbb{P}_{\otimes}^{f}\left[\left(Y_{\infty} \in B\right) \cap\left(\tau_{k}=\infty\right)\right]+\mathbb{P}_{\otimes}^{f}\left[\left(Y_{\infty} \in B\right) \cap\left(\tau_{k}<\infty\right)\right] \\
& \leqslant \mathbb{P}_{\otimes}^{f^{k}}\left[\left(Y_{\infty} \in B\right)\right]+\mathbb{P}\left[\left(\tau_{k}<\infty\right)\right] \\
& =\mathbb{P}\left[\int_{U}\left(\bar{D}_{u} f_{u}\right)^{2} \lambda^{-}(d u) \geqslant k\right] \stackrel{k \rightarrow \infty}{\longrightarrow} 0 .
\end{aligned}
$$

This concludes.

\subsection{The main anticipative result}

From now until the end of the paper we will assume for technical reasons that $f$ satisfies the additional assumption

$\left(\mathrm{H}^{\prime}\right): \quad f_{u}(\omega)=0 \quad$ for every $u: \omega(\{u\})=1$ 
which implies (HM). Note that if we apply Lemma 2.1 with $g_{u}(\omega):=\omega(\{u\})$, then we obtain that $\widetilde{\mathbb{P}}_{\omega}^{f}[\omega \cap \widetilde{\omega} \neq \emptyset]=0$ for every $\omega \in \Omega$; this means that the transformation only adds particles. We now turn to the anticipative part of the paper. We begin with an useful lemma.

Lemma 3.4. - Let $f$ a process satisfying $(\mathrm{H})$ and $\left(\mathrm{H}^{\prime}\right)$. Suppose that $f$ has a decomposition

$$
f=f^{1}+f^{2},
$$

where $f^{1}$ is a nonnegative process satisfying (L1) and $f^{2}$ is a non negative process such that the direct transformation associated to $f^{2}$ is absolutely continuous with respect to $\mathbb{P}$. Then we have (AC).

Remark. - This lemma roughly says that if we are in case of such an above decomposition, we do not have to worry about the $f^{1}$ part of $f$ - which gives an almost surely finite cloud - and the (AC) problem related to $f$ turns to an (AC)-type problem related to $f^{2}$, which may be simpler. Hence a finite perturbation of an absolute continuity situation is still an absolute continuity situation. Notice also that $f^{i}$ satisfies also $\left(\mathrm{H}^{\prime}\right)$ for $i \in\{1,2\}$ since they are nonnegative processes.

Proof. - We define for $i \in\{1,2\}$ the probability $\widetilde{\mathbb{P}}_{\omega}^{f^{i}} \in \mathcal{M}_{1}(\widetilde{\Omega}, \widetilde{\mathcal{F}})$ by (8), where we replace $f$ by $f^{i}$. We note

$$
L_{2}=\frac{d \mathbb{P}_{\otimes}^{f^{2}}}{d \mathbb{P}},
$$

which exists by our assumptions. The proof of the theorem will use two remarks.

Remark 1. - By using Theorem 3.1, $\tilde{\omega}$ is $\widetilde{\mathbb{P}}_{\omega}^{f^{1}}$-almost surely finite and has a density $\left\{Z_{\omega}^{f^{1}, \infty}(A), A \in P(U)\right\}$ with respect to the measure $|\lambda|$ on $P(U)$ for $\mathbb{P}$-almost every $\omega$. The additional assumption $\left(\mathrm{H}^{\prime}\right)$ implies that this density is in fact a density with respect to the measure $\lambda^{-}$on $P(U)$.

Remark 2.- Remember now that the sum of two independent Poisson variables is still a Poisson variable associated with the sum of the intensities; this remark leads to: for every $\omega \in \Omega$ and $G \in \mathcal{E}_{b}(\Omega)$, we have

$$
\widetilde{\mathbb{E}}_{\omega}^{f}[G(\tilde{\omega})]=\int_{\widetilde{\Omega}^{2}} G\left(\tilde{\omega} \cup \tilde{\omega}^{\prime}\right) \widetilde{\mathbb{P}}_{\omega}^{f^{1}}(d \tilde{\omega}) \widetilde{\mathbb{P}}_{\omega}^{f^{2}}\left(d \tilde{\omega}^{\prime}\right) .
$$

We now turn back to the proof of the lemma. We obtain by using Remark 1 that

$$
\begin{aligned}
\mathbb{E}_{\otimes}^{f}\left[F\left(Y_{\infty}\right)\right] & =\mathbb{E}\left[\int_{\widetilde{\Omega}}\left(\int_{\widetilde{\Omega}} F\left(\varepsilon_{\tilde{\omega} \cup \tilde{\omega}^{\prime}} \omega\right) \widetilde{\mathbb{P}}_{\omega}^{f^{2}}(d \tilde{\omega})\right) \widetilde{\mathbb{P}}_{\omega}^{f^{1}}\left(d \tilde{\omega}^{\prime}\right)\right] \\
& =\mathbb{E}\left[\int_{P(U)}\left(\int_{\widetilde{\Omega}} F\left(\varepsilon_{\tilde{\omega} \cup A} \omega\right) \widetilde{\mathbb{P}}_{\omega}^{f^{2}}(d \tilde{\omega})\right) Z_{\omega}^{f^{1}, \infty}(A) \lambda^{-}(d A)\right] .
\end{aligned}
$$


Observe now that, for any $\omega \in \Omega$ and $A \in P(U)$, then

$$
\widetilde{\mathbb{P}}_{\omega}^{f^{2}}[\tilde{\omega} \cap A \neq \emptyset]=0
$$

thanks to $\left(\mathrm{H}^{\prime}\right)$. Moreover, for any $A$ and $\tilde{\omega}$ such that $\tilde{\omega} \cap A=\emptyset$, we have that $\varepsilon_{\tilde{\omega} \cup A}=$ $\varepsilon_{A} \circ \varepsilon_{\tilde{\omega}}$. Therefore we finally come to

$$
\mathbb{E}_{\otimes}^{f}\left[F\left(Y_{\infty}\right)\right]=\int_{P(U)} \mathbb{E}_{\otimes}^{f^{2}}\left[F\left(\varepsilon_{A} Y_{\infty}\right) Z_{\omega}^{f^{1}, \infty}(A)\right] \lambda^{-}(d A)
$$

thanks to Fubini's theorem. Now denote by $\left\{G_{A}, A \in P(U)\right\}$ a process satisfying

$$
G_{A}\left(Y_{\infty}\right)=\mathbb{E}_{\otimes}^{f^{2}}\left[Z_{\omega}^{f^{1}, \infty}(A) \mid Y_{\infty}\right], \quad \forall A \in P(U) .
$$

We obtain that

$$
\begin{aligned}
\mathbb{E}_{\otimes}^{f}\left[F\left(Y_{\infty}\right)\right] & =\int_{P(U)} \mathbb{E}_{\otimes}^{f^{2}}\left[F\left(\varepsilon_{A} Y_{\infty}\right) G_{A}\left(Y_{\infty}\right)\right] \lambda^{-}(d A) \\
& =\int_{P(U)} \mathbb{E}\left[F\left(\varepsilon_{A} \omega\right) G_{A}(\omega) L_{2}\right] \lambda^{-}(d A) \\
& =\mathbb{E}\left[F\left(\int_{P(U)}\left(G_{A} L_{2}\right)^{\prime} \lambda^{+}(d A)\right)\right]
\end{aligned}
$$

by using Remark 2 for the second equality and the multiple Duality Formula of Proposition A.1 for the third one. This concludes.

The lemma we state now is a generalization of the last lemma, claiming (AC) when the process $f^{1}$ is no longer positive. The argument given for the last proof is then no longer applicable, since we cannot give a sense to a Poisson random measure whose intensity may eventually be negative. However, we can write the decomposition of $f^{1}$ into its positive/negative parts following

$$
f^{1}=f^{1,+}-f^{1,-} .
$$

The idea of the next lemma is the following: adding particles with an intensity $f$ is the same as considering two successive transformations: we first add particles with intensity $f^{1,+}+f^{2}$ and we then kill a finite number of particles with some rate $\breve{k}$ to compute. Those two steps will respectively use the results of the last lemma and Theorem 3.1.

LEMMA 3.5. - Suppose that $f$ satisfies $(\mathrm{H})$ and $\left(\mathrm{H}^{\prime}\right)$ and admits a decomposition of the form (24) where $f^{1}$ is a process satisfying $(\mathrm{H}),\left(\mathrm{H}^{\prime}\right)$ and $(\mathrm{L} 1)$ and $f^{2}$ is a nonnegative process satisfying

$$
\mathbb{P}_{\otimes}^{f^{2}} \ll \mathbb{P}
$$

Then we have (AC). 
Proof. - We fix for a moment $\omega$ such that

$$
\int_{U}\left|f_{u}^{1}(\omega)\right| \lambda^{-}(d u)<\infty
$$

and consider $\widehat{\mathbb{P}}_{\omega} \in \mathcal{M}_{1}(\Omega, \mathcal{F})$ such that $\lambda^{+}$is under $\widehat{\mathbb{P}}_{\omega}$ a Poisson random measure on $(U, \mathcal{U})$ with intensity

$$
\hat{\lambda}_{\omega}^{-}(.)=\left(f^{1,+}(\omega)+f_{.}^{2}(\omega)\right) \lambda^{-}(.) .
$$

We now define

$$
\check{k}_{u}^{\omega}=\left(f_{u}^{1,-}(\omega)\right) /\left(f_{u}^{2}(\omega)+f_{u}^{1,+}(\omega)\right) \in[0 ; 1]
$$

and the process $\check{f}^{\omega}: U \times \Omega \rightarrow[0 ; 1]$ given by

$$
\check{f}_{u}^{\omega}\left(\omega^{\prime}\right)=\omega^{\prime}(\{u\}) \check{k}_{u}^{\omega},
$$

which satisfies $(\mathrm{H})$. It may be seen that the direct transformation associated with $\check{f}^{\omega}$. kills a particle of an initial condition $\omega^{\prime}$ in $u$ with the deterministic rate $\breve{k}_{u}^{\omega}$-remember that $\omega$ is fixed - and almost surely never adds particles. Remark that

$$
\begin{aligned}
& \int_{\Omega}\left(\int_{U} \check{f}_{u}^{\omega}\left(\omega^{\prime}\right)\left(\omega^{\prime}(d u)+\hat{\lambda}_{\omega}^{-}(d u)\right)\right) \widehat{\mathbb{P}}_{\omega}\left(d \omega^{\prime}\right) \\
& =\widehat{\mathbb{E}}_{\omega}\left[\int_{U} \check{k}_{u}^{\omega} \omega^{\prime}(d u)\right]+\widehat{\mathbb{E}}_{\omega}\left[\int_{U} \check{f}_{u}^{\omega}\left(\omega^{\prime}\right) \hat{\lambda}_{\omega}^{-}(d u)\right]
\end{aligned}
$$

and it may be easily seen that the second expectation is null since $\hat{\lambda}_{\omega}^{-}$is a diffuse measure. Hence the last expression is equal to

$$
\int_{U} \check{k}_{u}^{\omega} \hat{\lambda}_{\omega}^{-}(d u)=\int_{U} f_{u}^{1,-}(\omega) \lambda^{-}(d u)<\infty .
$$

This implies that $\check{f}^{\omega}$ satisfies (L1) with $\mathbb{P}:=\widehat{\mathbb{P}}_{\omega}$ and $\lambda^{-}(d u):=\hat{\lambda}_{\omega}^{-}(d u)$. Consequently, by using Theorem 3.1 in the setup of the Poisson space $\left(\Omega, \mathcal{F}, \widehat{\mathbb{P}}_{\omega}\right)$, we obtain that there exists a positive, measurable $\breve{L}^{\omega} \in L^{1}\left(\Omega, \mathcal{F}, \widehat{\mathbb{P}}_{\omega}\right)$ such that for every $F \in \mathcal{E}_{b}(\Omega)$ :

$$
\int_{\omega^{\prime} \in \Omega}\left(\int_{\widetilde{\Omega}} F\left(\varepsilon_{\tilde{\omega}} \omega^{\prime}\right) \widetilde{\mathbb{P}}_{\omega^{\prime}}^{\check{f}^{\prime}}(d \tilde{\omega})\right) \widehat{\mathbb{P}}_{\omega}\left(d \omega^{\prime}\right)=\widehat{\mathbb{E}}_{\omega}\left[F \check{L}^{\omega}\right] .
$$

On the other hand, we can show by standard arguments that $\omega^{\prime}$ is under $\check{L}^{\omega} \widehat{\mathbb{P}}_{\omega}\left(d \omega^{\prime}\right)$ a Poisson random measure with intensity

$$
\left(1-\check{k}_{.}^{\omega}\right) \hat{\lambda}_{\omega}(.)=\left(f^{2}(\omega)+f^{1,+}(\omega)-f^{1,-}(\omega)\right) \lambda^{-}(.)=f .(\omega) \lambda^{-}(.) .
$$

This roughly says that if you kill the particles of a Poisson random measure with intensity $m(d u)$ with a deterministic rate $k$, one independently of each others, then the final system 
has the distribution of a random Poisson measure with intensity $(1-k) m(d u)$. This remark implies that for every $F \in \mathcal{E}_{b}(\Omega)$, then

$$
\int_{\Omega} F\left(\omega^{\prime}\right) \check{L}^{\omega}\left(\omega^{\prime}\right) \widehat{\mathbb{P}}_{\omega}\left(d \omega^{\prime}\right)=\widetilde{\mathbb{E}}_{\omega}^{f}[F(\tilde{\omega})]
$$

where $\tilde{\omega} \in \widetilde{\Omega}$ is identified to an element of $\Omega$ in the last expression. All what we told until now is true for $\omega$ satisfying (25), that is, for $\mathbb{P}$-almost every $\omega$ thanks to the hypothesis. Let us now introduce the probability $\widehat{P}_{\otimes} \in \mathcal{M}_{1}\left(\Omega^{2}, \mathcal{F} \otimes \mathcal{F}\right)$ defined by

$$
\widehat{P}_{\otimes}\left(d \omega^{\prime}, d \omega\right)=\widehat{\mathbb{P}}_{\omega}\left(d \omega^{\prime}\right) \mathbb{P}(d \omega) .
$$

An application of Lemma 3.4 with $f:=f^{1,+}+f^{2}$ gives that

$$
\widehat{P}_{\otimes} \circ\left(\varepsilon_{\omega^{\prime}} \omega\right)^{-1} \ll \mathbb{P}
$$

and we note $L$ the associated density. Let $G$ a random variable such that

$$
G\left(\varepsilon_{\omega^{\prime}} \omega\right)=\widehat{E}_{\otimes}\left[\check{L}^{\omega}\left(\omega^{\prime}\right) \mid \varepsilon_{\omega^{\prime}} \omega\right],
$$

we conclude by using (26) and then (27) following

$$
\begin{aligned}
\mathbb{E}_{\otimes}^{f}\left[F\left(Y_{\infty}\right)\right] & =\mathbb{E}\left[\int_{\Omega} F\left(\varepsilon_{\omega^{\prime}} \omega\right) \check{L}^{\omega}\left(\omega^{\prime}\right) \widehat{\mathbb{P}}_{\omega}\left(d \omega^{\prime}\right)\right] \\
& =\widehat{E}_{\otimes}\left[F\left(\varepsilon_{\omega^{\prime}} \omega\right) G\left(\varepsilon_{\omega^{\prime}} \omega\right)\right] \\
& =\mathbb{E}[F(G L)] .
\end{aligned}
$$

It is over.

The main result is an exploitation of the above Lemma 3.5 in the case of an adapted process $f^{2}$, whose related absolute continuity problem is solved thanks to Theorem 3.2.

THEOREM 3.3 (Anticipative theorem). - Assume that $f$ satisfies $(\mathrm{H})$ and $\left(\mathrm{H}^{\prime}\right)$ and has a decomposition

$$
f=f^{1}+f^{a}
$$

where $f^{1}$ is a process satisfying $(\mathrm{H}),\left(\mathrm{H}^{\prime}\right)$ and $(\mathrm{L} 1)$ and $f^{a}$ is a nonnegative adapted process satisfying (L2). Then we have (AC).

The proof is a direct implication of Lemma 3.5 and of the adapted Theorem 3.2. It tells that a system perturbated by an adapted cloud - which may be infinite - and an independent, almost surely finite anticipative cloud has the wished absolute continuity property. We give now an example. Remember that our notion of adaptation is related with the initial choice of our direction; the fact that it is a choice may be useful. 


\section{Example and sufficient conditions to solve the original problem}

We study in this section some examples of applications of the anticipative Theorem 3.3 in the particular setup of the canonical example of Section 1.2. in this setup, we have $U=[0 ; 1] \times\left(\mathbb{R}^{d} \backslash\{0\}\right)$ for some $d \in \mathbb{N}^{*}$. This space is equipped with a measure $\lambda^{-}$having the product form $d t \mu(d x)$ for some $\sigma$-finite, positive measure on $\mathbb{R}^{d}$ satisfying (6). The direction is chosen following

$$
U_{t}=\{[0 ; 1] \times\{x:|x| \geqslant 1 / t\}\}
$$

and we can define a Lévy process $X:[0 ; 1] \times \Omega \rightarrow \mathbb{R}^{d}$ with Lévy measure $\mu$ following (7). We are interested with a particular type of applications: let give us a bounded, measurable mapping $\hat{f}: U \times \mathbb{R}^{d} \rightarrow \mathbb{R}^{+}$. We consider a process $Z:[0 ; 1] \times$ $\Omega \rightarrow \mathbb{R}^{d}$ and define an intensity process $f: U \times \Omega \rightarrow \mathbb{R}^{+}$following

$$
f_{u}(\omega)=\hat{f}_{u}\left(Z_{1}(\omega)\right)(1-\omega(u)) .
$$

It obviously fulfills $\left(\mathrm{H}^{\prime}\right)$ and, by assuming that $\hat{f}$ is bounded on each $U_{t}$, it satisfies also $(\mathrm{H})$. The question that we will investigate in this section is: let $Z$ be a given process, can we find sufficient conditions on $\hat{f}$ to have that the direct transformation associated with the process $f$ given by (28) satisfies the (AC) problem?

The study of this example is divided into three parts. The first one gives a sufficient condition for a general process $Z$, up to some restrictions on $\hat{f}$. In the second part Section 4.2, we will study the sophisticated example of a process $Z$ which is the solution of an SDE driven by the jumps of the Lévy process $X$ given by (7) and we will deduce a sufficient condition thanks to the anticipative Theorem 3.3. For sake of clearness, this section is made in a $L^{2}$ framework. In the last Section 4.3 we turn back to the original problem of our introduction and find some sufficient conditions to solve it in a larger $L^{p}$ context for $p \geqslant 1$.

\subsection{General framework of the example}

From now, $C$ will denote a finite, positive constant which may change from one line to another. We do not assume here that $Z$ has a special shape and then we have to deal with a direct, a priori anticipative transformation. Hence we want to use the anticipative Theorem 3.3 and find a decomposition of $f$ into a positive, adapted process satisfying the (L2) assumption and a process $f^{1}$ satisfying (L1).

We assume that there exists a collection $\left(Z^{t}\right)_{t \in \mathbb{R}^{+}}$of processes on $[0 ; 1] \times \Omega$ satisfying

(i) for every $t, Z_{1}^{t}$ is a $\mathcal{F}_{t}$-measurable random variable,

(ii) $(t, \omega) \mapsto Z_{1}^{t}(\omega)$ is measurable.

We outline that $\left(Z^{t}\right)_{t}$ may be arbitrary chosen provided that it satisfies both last points. We define

$$
f_{s x}^{a}(\omega)=\hat{f}_{s x}\left(Z_{1}^{1 /|x|}(\omega)\right)(1-\omega(u)) .
$$

It is obvious that it is a non negative, $\left(\mathcal{F}_{t}\right)_{t}$-adapted process since the gauge function $G$ of the direction $\left(U_{t}\right)_{t}$ is given by $G(s, x)=1 /|x|$. Let us introduce a first assumption 
on $\hat{f}$ :

$$
u \mapsto\left(\sup _{x}\left|\hat{f}_{u}(x)\right|\right) \in L^{2}\left(U, \mathcal{U}, \lambda^{-}\right) .
$$

This assumption implies that $f^{a}$ fulfills the (L2) assumption. We now define $f^{1}=$ $f-f^{a}$ which obviously satisfies $(\mathrm{H}),\left(\mathrm{H}^{\prime}\right)$ and aim at finding sufficient condition to ensure that it satisfies (L1). Notice that this process has no reason to be non negative and this illustrates why Lemma 3.5 is actually an improvement of Lemma 3.4. We introduce a new Lipschitz assumption on $\hat{f}$ : we assume that there exists a measurable mapping $\hat{c}$ on $U$ such that for every $u$ in $U, \hat{f}_{u}$ is a Lipschitz function with constant $\hat{c}(u)$. We will assume that $\hat{c}$ satisfies the (p-Lip) assumption given by

$$
\text { (p-Lip): } \hat{c} \in L^{p}\left(U, \lambda^{-}\right)
$$

for some $p \geqslant 1$. In the following, $q$ will denote the conjugate of $p$ given by

$$
\frac{1}{p}+\frac{1}{q}=1
$$

Thanks to Hölder's inequality, we obtain that $f^{1}$ satisfies (L1) if

$$
\int_{\mathbb{R}^{d}} \mathbb{E}\left[\left|Z_{1}-Z_{1}^{1 /|x|}\right|^{q}\right] \mu(d x)<\infty .
$$

In the two following examples of Sections 4.2 and 4.3, we will give us the process $Z$. By choosing a good collection $\left(Z^{t}\right)_{t}$, we will give sufficient conditions for the (AC) problem by proving that (30), (p-Lip) and (31) assumptions are in force for some $p \geqslant 1$.

\subsection{The case of $Z$ satisfying an SDE}

In this example, $Z$ is a solution to a SDE driven by the jumps of $X$ : we give us two measurable mappings $g: U \times \mathbb{R}^{d} \rightarrow \mathbb{R}^{d}$ and $h:[0 ; 1] \times \mathbb{R}^{d} \rightarrow \mathbb{R}^{d}$ such that:

(i) There exists $c \in L^{2}\left(U, \lambda^{-}\right)$such that for every $(u, a, b) \in U \times \mathbb{R}^{d} \times \mathbb{R}^{d}$, then

$$
|g(u, a)-g(u, b)| \leqslant c(u)|a-b|,
$$

where |.| stands for the Euclidean norm in $\mathbb{R}^{d}$.

(ii) There exists $d \in L^{1}([0 ; 1], d t)$ such that for every $(s, a, b) \in U \times \mathbb{R}^{d} \times \mathbb{R}^{d}$, then

$$
|h(s, a)-h(s, b)| \leqslant d(s)|a-b| .
$$

(iii) $\int_{U}|g|^{2}(u, 0) \lambda^{-}(d u)<\infty$ and $\int_{0}^{1}|h|(s, 0) d s<\infty$.

We consider the SDE given by

$(E): \quad Z_{r}(\omega)=1+\int_{0}^{r} \int_{x \in E} g\left(s, x, Z_{s^{-}}\right) \lambda(\omega, d s, d x)+\int_{0}^{r} h\left(s, Z_{s^{-}}\right) d s, \quad r \in[0 ; 1]$. 
Under the assumptions (i) and (ii), one can show by a Picard iteration that this equation has an unique càd làg solution satisfying

$$
\mathbb{E}\left[\sup _{0 \leqslant s \leqslant 1}\left|Z_{s}\right|^{2}\right] \leqslant C<\infty .
$$

Let give us $\hat{f}$. The question is to solve the (AC) problem related with the process $f$ given by (28). We will here restrict our study to the case of $\hat{f}$ satisfying (30) and checking (2-Lip). For $t \in \mathbb{R}^{+}$we introduce the process $Z^{t}:[0 ; 1] \times \Omega \rightarrow \mathbb{R}^{d}$ solution to the equation

$$
Z_{r}^{t}(\omega)=1+\int_{0}^{r} \int_{|x| \geqslant \frac{1}{t}} g\left(s, x, Z_{s^{-}}^{t}\right) \lambda(\omega, d s, d x)+\int_{0}^{r} h\left(s, Z_{s^{-}}^{t}\right) d s, \quad r \in[0 ; 1] .
$$

Notice that for every $r, t$, the random variable $Z_{r}^{t}$ is $\mathcal{F}_{t}$-measurable, that is, it depends of the jumps of $X$ having a size greater than $1 / t$. One sees easily that such a collection $\left(Z^{t}\right)_{t}$ satisfies the points (i) and (ii) of Section 4.1 and we finally define $f^{a}$ by (29). The last thing to prove is the (31) sufficient condition in the case of $q=2$. Let $r \in[0 ; 1]$ and $t \in \mathbb{R}^{+}$; we set

$$
V_{r}^{t}=\mathbb{E}\left[\left|Z_{r}-Z_{r}^{t}\right|^{2}\right]
$$

we have

$$
\begin{aligned}
V_{r}^{t} \leqslant & C\left(\mathbb{E}\left[\left|\int_{\left([0 ; r] \times \mathbb{R}^{d}\right) \cap U_{t}}\left(g\left(s, x, Z_{s^{-}}\right)-g\left(s, x, Z_{s^{-}}^{t}\right)\right) \lambda(\omega, d s, d x)\right|^{2}\right]\right. \\
& \left.+\mathbb{E}\left[\left|\int_{\left([0 ; r] \times \mathbb{R}^{d}\right) \backslash U_{t}} g\left(s, x, Z_{s^{-}}\right) \lambda(\omega, d s, d x)\right|^{2}\right]+\mathbb{E}\left[\left(\int_{0}^{r} d(s)\left|Z_{s}-Z_{s}^{t}\right| d s\right)^{2}\right]\right) \\
= & C\left(E_{1}+E_{2}+E_{3}\right)
\end{aligned}
$$

where $E_{1}$ to $E_{3}$ are the respective expectations of the above sum. By using the isometric nature of the Ito stochastic integral and the Lipschitz assumption (i) we obtain that

$$
\begin{aligned}
E_{1} & =\int_{\left([0 ; r] \times \mathbb{R}^{d}\right) \cap U_{t}} \mathbb{E}\left[\left|g\left(s, x, Z_{s^{-}}\right)-g\left(s, x, Z_{s^{-}}^{t}\right)\right|^{2}\right] \lambda^{-}(d s, d x) \\
& \leqslant \int_{\left([0 ; r] \times \mathbb{R}^{d}\right) \cap U_{t}} c^{2}(s, x) \mathbb{E}\left[\left|Z_{s^{-}}-Z_{s^{-}}^{t}\right|^{2}\right] \lambda^{-}(d s, d x) \\
& =\int_{0}^{r}\left(\int_{\{x:|x| \geqslant 1 / t\}} c^{2}(s, x) \mu(d x)\right) V_{s}^{t} d s .
\end{aligned}
$$

By using (i) and (iii) we obtain that 


$$
\begin{aligned}
E_{2} \leqslant & C\left(\int_{0}^{r}\left(\int_{\{x: 0<|x|<1 / t\}} c^{2}(s, x) \mu(d x)\right) \mathbb{E}\left[\sup _{0 \leqslant s \leqslant 1}\left|Z_{s}\right|^{2}\right] d s\right. \\
& \left.+\int_{[0 ; 1] \times\{x: 0<|x|<1 / t\}}|g(u, 0)|^{2} \lambda^{-}(d u)\right) \\
\leqslant & C P(1 / t)
\end{aligned}
$$

where for any $T \in \mathbb{R}^{+}$we note

$$
P(T)=\left(\int_{[0 ; 1] \times\{x: 0<|x|<T\}}\left(c^{2}(s, x)+|g(s, x, 0)|^{2}\right) d s \mu(d x)\right) .
$$

By using the Cauchy-Schwarz inequality with respect to the finite measure $d(s) d s$ on $[0 ; 1]$ we obtain that

$$
E_{3} \leqslant C \int_{0}^{r} d(s) V_{s}^{t} d s
$$

We deduce from those estimations that for every $r \in[0 ; 1]$ we have

$$
V_{r}^{t} \leqslant C P(1 / t)+C \int_{0}^{r}\left(d(s)+\int_{\mathbb{R}^{d}} c^{2}(s, x) \mu(d x)\right) V_{s}^{t} d s
$$

and by a Gronwall argument this gives by using the assumptions on $c$ and $d$ that

$$
V_{1}^{t} \leqslant C P(1 / t)
$$

Finally, the sufficient condition (31) giving (AC) is implied by

$$
\int_{\mathbb{R}^{d}} P(|x|) \mu(d x)<\infty .
$$

We give a short example in which we may explicit the above sufficient condition. We consider that $\mu$ has the form

$$
\mu(d x)=1_{\{|x| \leqslant 1\}} \frac{d x}{|x|^{d+\beta}}
$$

for some $\beta \in] 0 ; 2[$. In this case, the process $X$ given by (7) is a truncated, $\beta$-stable isotropic Lévy process. We assume moreover that $d \equiv 0$ and

$$
\sup _{s}|c(s, x)|+\sup _{s}|g(s, x, 0)| \leqslant C|x|^{\alpha}
$$


for some $\alpha \in \mathbb{R}$. The assumptions (i) and (iii) of the beginning of this section are in force if

$$
\alpha>\frac{\beta}{2} .
$$

On the other hand a short computation gives that

$$
P(|x|) \leqslant C(|x| \wedge 1)^{2 \alpha-\beta}
$$

and then (33) is in force if

$$
\alpha>\beta .
$$

\subsection{The case of $Z=X$ : coming back to the original problem}

Let us come back to our original motivation exposed in the introduction and we will here use the notation of this first paragraph of the paper. Notice that one may chose the triplet $(\Omega, \mathcal{F}, \mathbb{P})$ equal to the Poisson space of the canonical example with a measure $\mu$ given by

$$
\mu(d x)=\frac{d x}{|x|^{d+\beta}}
$$

and the process $X$ may be chosen as the one given by (7): it is a Lévy, isotropic and $\beta$-stable process. We now show how some sufficient conditions to this original problem may be deduced from our study of the (AC)-type problem examinated in Section 4.1: thanks to Proposition 4.1, we will simulate the perturbation of the paths of $X .(\omega)$ by the path of $\Psi\left(X_{1}(\omega)\right) X^{\prime}\left(\omega^{\prime}\right)$ thanks to an anticipative perturbation of the Poisson space by a direct transformation associated with some process $f$. This intensity $f$ will have the form (28) with $Z \equiv X$ therefore we will use the preliminary results of Section 4.1.

Let us note

$$
\mu^{\prime}(d x)=\frac{d x}{|x|^{d+\beta^{\prime}}}
$$

the Lévy measure of $X^{\prime}$. We will assume that $0<\beta^{\prime}<1$ and that $\Psi$ is a bounded, Lipschitz function for the rest of the paper. The assumption on $\beta^{\prime}$ implies that $X^{\prime}$ is a finite variation process and in fact it is the convergent sum of its jumps. The distribution of the process $X^{\prime}$ is defined by the characteristic function

$$
\mathbb{E}^{\prime}\left[\exp \left(i\left\langle\lambda, X_{1}^{\prime}\right\rangle\right)\right]=\exp \left(\int_{\mathbb{R}^{d}}\left(\mathrm{e}^{i\langle\lambda, x\rangle}-1\right) \mu^{\prime}(d x)\right)
$$

for every $\lambda \in \mathbb{R}^{d}$. Let $\omega \in \Omega$. For $s \in \mathbb{R}^{+}$we denote by $H(s)$ the scalar multiplicator by $s$ on $\mathbb{R}^{d}$. It is obvious that for every $z \in \mathbb{R}^{d}$, the process $\left\{\Psi(z) \times X_{t}^{\prime}, t \in[0 ; 1]\right\}$ is under $\mathbb{P}^{\prime}$ a Lévy process with a Lévy measure equal to

$$
\mu_{z}^{\prime}=\mu^{\prime} \circ[H(\Psi(z))]^{-1} .
$$


Now we define the measure $\lambda_{z}^{-}$on $U$ by $\lambda_{z}^{-}(d s, d x)=d s \mu_{z}^{\prime}(d x)$ and for $z \in \mathbb{R}^{d}$ we define $\hat{f} .(z)$ following

$$
\hat{f}_{s x}(z)=\frac{\lambda_{z}^{-}(d s, d x)}{\lambda^{-}(d s, d x)}=|\Psi(z)|^{\beta^{\prime}} \times|x|^{\beta-\beta^{\prime}} .
$$

We then define the process $f$ by (28) with $Z \equiv X$. The next proposition links the original problem and the (AC) problem - the process $\bar{X}$ is given in the first part of the introduction:

PROPOSITION 4.1. - Then we have the following distribution equality:

$$
\mathbb{P}_{\otimes}^{f} \circ\left\{X_{t}\left(\varepsilon_{\tilde{\omega}} \omega\right), t \in[0 ; 1]\right\}^{-1}=\mathbb{P}^{\prime} \circ\left\{\bar{X}_{t}, t \in[0 ; 1]\right\}^{-1} .
$$

In particular, sufficient conditions to the original problem may be given by the sufficient conditions to the (AC) problem of Section 4.1. This proposition may be quickly proven since conditionally on $\omega$, we add in the right side a finite variation Lévy process having a Lévy measure $\mu_{\Psi_{1}(\omega)}^{\prime}$. In the left side, we include - and never remove, thanks to the $\left(\mathrm{H}^{\prime}\right)$ assumption - the particles of a Poisson random measure $\tilde{\omega}$ associated with the intensity

$$
\lambda_{\Psi_{1}(\omega)}^{-}(d s, d x)=d s \mu_{\Psi_{1}(\omega)}^{\prime}(d x)
$$

in the set

$$
\left\{(s, x) \in[0 ; 1] \times\left(\mathbb{R}^{d} \backslash\{0\}\right): \Delta X_{s}(\omega)=x\right\} .
$$

This is the same than adding to the path $X .(\omega)$ the jumps of a (finite variation) Lévy process having a Lévy measure $\mu_{\Psi_{1}(\omega)}^{\prime}$ and this concludes. Observe now that for every $\omega$

$$
\int_{(s, x):|x|>1} f_{s x}(\omega) \lambda^{-}(d s, d x)<\infty .
$$

By using Lemma 3.4 with

$$
f_{s x}^{1}:=1_{\{|x|>1\}} f_{s x}
$$

and $f^{2}:=f-f^{1}$ we obtain that the (AC) problem may be reduced to the case of a truncated $f:=f^{2}$ : this philosophically tells that the large jumps of $X^{\prime}-$ which are almost surely finite - are not involved in the absolute continuity question and from now we study the (AC) problem associated with $f:=f^{2}$, which is null if $|x|>1$. This comes back to consider that $\hat{f}_{s x}$ and its Lipschitz factor $\hat{c}(s, x)$ are null if $|x|>1$. For $t \in \mathbb{R}^{+}$, we introduce the process:

$$
Z_{s}^{t}(\omega)=\int_{[0 ; s] \times\{1 / t \leqslant|x| \leqslant 1\}} x \lambda(\omega, d s, d x)+\int_{[0 ; s] \times\{|x|>1 \vee(1 / t)\}} x \lambda^{+}(\omega, d s, d x) .
$$

We will use the results of Section 4.1 in the following order: 
- We satisfythat the sequence $\left(Z^{t}\right)_{t}$ given by (39) satisfies both points (i) and (ii) of Section 4.1.

- We give sufficient conditions on $\Psi, \beta$ and $\beta^{\prime}$ to have the condition (30).

- We give sufficient conditions on the same objects to have the (p-Lip) assumption for some $p \geqslant 1$.

- We discuss the last condition (31) with the values of $q$ given by the last point. The first point is easily checked.

Second point. A simple computation shows that (30) is in force if

$$
\beta^{\prime}<\frac{\beta}{2}
$$

since $\Psi$ is bounded.

Third point. We now have for which values of $p$ the Lipschitz factor $\hat{c}$ of $\hat{f}$ is in $L^{p}\left(U, \lambda^{-}\right)$. We now assume that

$$
z \mapsto|\Psi(z)|^{\beta^{\prime}} \text { is a Lipschitz function. }
$$

A simple computation shows that one can set

$$
\hat{c}(s, x)=C|x|^{\beta-\beta^{\prime}} 1_{\{|x| \leqslant 1\}} .
$$

Hence $\hat{c}$ satisfies (p-Lip) if

$$
p>\frac{\beta}{\beta-\beta^{\prime}} .
$$

Note that (40) implies that the right side term is smaller than 2.

Fourth point. Notice that, thanks the truncation argument (38), the integral of the last condition (31) shall be considered on the domain $\{(s, x):|x| \leqslant 1\}$. For $|x| \leqslant 1$ we have

$$
\begin{aligned}
\mathbb{E}\left[\left|Z_{1}-Z_{1}^{1 /|x|}\right|^{q}\right] & =\mathbb{E}\left[\left|\int_{[0 ; 1] \times\{|y| \leqslant|x|\}} y \lambda(\omega, d s, d y)\right|^{q}\right] \\
& \leqslant C \mathbb{E}\left[\left(\int_{[0 ; 1] \times\{|y| \leqslant|x|\}}|y|^{2} \lambda^{+}(\omega, d s, d y)\right)^{q / 2}\right]
\end{aligned}
$$

by using the Burkholder-Davis-Gundy estimates for the last inequality. We use Lemma 5.1 with $a:=1, b:=q / 2$ and $f(s, x)=|x|^{2}$ to estimate the above expectation. We obtain that it is smaller than -[]$_{2}$ is given by (A.2) and we convent that $\sum_{0}^{-1}(\ldots)=0$ :

$$
\begin{aligned}
& C\left(\sum_{k=0}^{[q / 2]_{2}-1}\left(\int_{\{|y| \leqslant|x|\}}|y|^{2^{k+1}} \mu(d y)\right)^{\frac{q}{2^{k+1}}}+\int_{\{|y| \leqslant|x|\}}|y|^{q} \mu(d y)\right) \\
& =C\left(\left(\sum_{k=0}^{[q / 2]_{2}-1}|x|^{\left(1-\frac{\beta}{2^{k+1}}\right) \times q}\right)+|x|^{q-\beta}\right)
\end{aligned}
$$


provided that $q>\beta$ to ensure the existence of the above integrals. A simple computation of those terms shows that the necessary condition (31) giving (AC) is satisfied if

$$
\beta<\left(\frac{2 q}{q+2} \wedge \frac{q}{2}\right) .
$$

Conclusion. Remind that we are free to chose $p$ provided that it satisfies (42) and we actually have to do the best choice implying that (43) is the weakest possible, that is, the best value of $p$ to have the biggest right side term of (43). Note that the functions of $q$ of the right side of this relation are continuous, increasing functions. On the other hand we have

$$
p \downarrow \frac{\beta}{\beta-\beta^{\prime}} \quad \Leftrightarrow \quad q \uparrow \frac{\beta}{\beta^{\prime}}>2,
$$

the last inequality is due to (40). This implies that

$$
\frac{2 q}{q+2} \uparrow \frac{2 \beta}{\beta+2 \beta^{\prime}} \quad \text { and } \quad \frac{q}{2} \uparrow \frac{\beta}{2 \beta^{\prime}} \text {. }
$$

By using those approximations in the (40) and (43) relations, we respectively obtain the relations

$$
\beta^{\prime}<\frac{\beta}{2}, \quad \beta<2\left(1-\beta^{\prime}\right), \quad \beta^{\prime}<\frac{1}{2} .
$$

It may be easily seen that the two first conditions imply the third one if $\beta \in] 0 ; 2[$. We formulate all the work of this section in the following proposition.

PROPOSITION 4.2 (Sufficient conditions for the original problem). - Assume that $\Psi: \mathbb{R}^{d} \rightarrow \mathbb{R}$ is a bounded function satisfying the Lipschitz assumption (41). Assume that

$$
\beta^{\prime}<\left(\frac{\beta}{2}\right) \wedge\left(1-\frac{\beta}{2}\right),
$$

then the original problem is solved and we have the wished absolute continuity result.

We conclude by some comments:

- in an adapted context, for instance if $\Psi$ is a constant function, the only needed condition is the first half (40) of (44) thanks to the adapted Theorem 3.2. It should be possible that it is also a necessary condition in this case.

- The domain of $\beta$ for which our conditions are the best possible is for $\beta \in] 0 ; 1]$. In this case, the (44) system turn simply to (40), as in the adapted case.

- The second part of the (44) condition is a purely anticipative one. It is a direct consequence of our method, namely of the necessity to have an almost surely finite anticipative part of the cloud. It illustrates the limit of our method because the closer we are to the Brownian case $\beta=2$, the smaller $\beta^{\prime}$ should be. It may be possible that this condition could be relaxed. 


\section{Appendix A}

\section{A.1 Duality Formulae}

We give here without proof the Duality Formulae of [10].

PROPOSITION A.1 (Duality Formula).- Single version: Let $Z: U \times \Omega \rightarrow \mathbb{R} a$ positive process, then

$$
\mathbb{E}\left[\int_{U} z_{u}|\lambda|(\omega, d u)\right]=\mathbb{E}\left[\int_{U} z_{u} \circ \varepsilon_{u}|\lambda|(\omega, d u)\right] .
$$

Multiple version: Let $Z_{A}, A \in P(U)$ be a positive process; we denote by $Z_{A}^{\prime}$ the $P(U)$-indexed process $Z_{A} \circ \varepsilon_{A}$. Let $\Phi$ the mapping from $P(U) \times \Omega$ in itself defined by $\Phi(A, \omega)=\left(A, \varepsilon_{A} \omega\right)$. Then we have

$$
\int_{P(U) \times \Omega}(G \circ \Phi)(A, \omega) Z_{A}(\omega) v(d A, d \omega)=\int_{P(U) \times \Omega} G(A, \omega) Z_{A}^{\prime}(\omega) v(d A, d \omega) .
$$

By using the single version, one easily satisfies that for every $|\lambda|$-integrable processes $z$ and $g$, one has

$$
\mathbb{E}\left[\int_{U} g_{u} \bar{D}_{u} z_{u}|\lambda|(\omega, d u)\right]=\mathbb{E}\left[\int_{U} \bar{D}_{u} g_{u} z_{u}|\lambda|(\omega, d u)\right] .
$$

\section{A.2 Moment estimation}

We will here use that for any $\alpha \leqslant 1$ we have

$$
\left(\sum_{i} x_{i}\right)^{\alpha} \leqslant\left(\sum_{i} x_{i}^{\alpha}\right)
$$

for any sum of positive $\left(x_{i}\right)_{i}$. For $q \in \mathbb{R}^{+}$, we note

$$
[q]_{2}=\inf \left\{n \in \mathbb{N}: q / 2^{n} \leqslant 1\right\} .
$$

LEMMA A.1. - Let $f: U \rightarrow \mathbb{R}^{+}$a measurable mapping. Let us note for $a, b \in \mathbb{R}^{+}$

$$
I^{ \pm}(a, b)=\mathbb{E}\left[\left(\int_{U} f^{a}(u) \lambda^{ \pm}(d u)\right)^{b}\right] .
$$

Then - we convent that $\sum_{0}^{-1}(\ldots)=0$ :

$$
I^{+}(a, b) \leqslant C\left(\sum_{k=0}^{[b]_{2}-1} I^{-}\left(2^{k} a, \frac{b}{2^{k}}\right)+I^{-}(a b, 1)\right) .
$$


Proof. - We have thanks to the Burkholder-Davis-Gundy estimations that for any $a, b \in \mathbb{R}^{+}$,

$$
\begin{aligned}
I^{+}(a, b) & \leqslant C\left(\mathbb{E}\left[\left(\int_{U} f^{a}(u) \lambda(\omega, d u)\right)^{b}\right]+I^{-}(a, b)\right) \\
& \leqslant C\left(I^{+}(2 a, b / 2)+I^{-}(a, b)\right) .
\end{aligned}
$$

Observe now that for any $c \in \mathbb{R}^{+}$and $d \in[0 ; 1]$ we have

$$
I^{+}(c, d) \leqslant I^{+}(c d, 1)=I^{-}(c d, 1) .
$$

This is a direct consequence of (A.1). The result is obtained by iterating (A.3) until having the second exponent of the $I^{+}$term smaller than 1 . One concludes with (A.4).

\section{REFERENCES}

[1] R. Buckdahn, Anticipative Girsanov transformation, Probab. Theory Related Fields 89 (1991) 211-238.

[2] R. Buckdahn, O. Enchev, Nonlinear transformations on the abstract Wiener space, Tech. Report 240 (Neue Folge), Humboldt Univ., Berlin, 1989.

[3] R.H. Cameron, W.T. Martin, Transformation of Wiener integral under translation, Ann. Math. 45 (1944) 386-396.

[4] O. Enchev, D.W. Stroock, Anticipative diffusions and related change of measure, J. Funct. Anal. 116 (1996) 449-477.

[5] I. Gyøngy, Mimicking complicated stochastic differential equations by simpler ones, Preprint.

[6] J. Jacod, A.N. Shiryaev, Limit Theorems for Stochastic Processes, Springer-Verlag, 1984.

[7] F. Nicaise, Anticipative Markovian transformations on the Poisson space, Stochastic Processes and their Applications, to appear.

[8] D. Nualart, The Malliavin Calculus and Related Topics, Springer-Verlag, 1995.

[9] D. Nualart, J. Vives, Anticipative calculus for the Poisson process based on the Fock space, in: Séminaire de Probabilité XXIV, Lect. Notes in Math. Vol.

[10] J. Picard, Formules de dualité sur l'espace de Poisson, Ann. Inst. Henri Poincaré 32 (4) (1996) 509-548.

[11] J. Picard, Transformations et équations anticipantes pour les processus de Poisson, Ann. Math. Blaise Pascal 3 (1) (1996) 111-123.

[12] A.S. Ustunel, M. Zakai, Transformation of Measure on Wiener Space, Springer-Verlag, 2000.

[13] A.S. Ustunel, M. Zakai, Transformation of the Wiener measure under anticipative flows, Probab. Theory Related Fields 93 (1992) 91-136. 